\title{
Experimental Searches for Dark Matter
}

\author{
Timothy J. Sumner \\ Astrophysics Group, Blackett Laboratory, Imperial College \\ Prince Consort Road, London SW7 2BZ \\ e-mail:t.sumner@ic.ac.uk \\ http://astro.ic.ac.uk \\ Published on 23 July 2002 \\ www.livingreviews.org/Articles/Volume5/2002-4sumner \\ Living Reviews in Relativity \\ Published by the Max Planck Institute for Gravitational Physics \\ Albert Einstein Institute, Germany
}

\begin{abstract}
There is now an enormously rich variety of experimental techniques being brought to bear on experimental searches for dark matter, covering a wide range of suggested forms for it. The existence of "dark matter", in some form or other, is inferred from a number of relatively simple observations and the problem has been known for over half a century. To explain "dark matter" is one of the foremost challenges today - the answer will be of fundamental importance to cosmologists, astrophysicists, particle physicists, and general relativists. In this article, I will give a brief review of the observational evidence (concentrating on areas of current significant activity), followed by anequally brief summary of candidate solutions for the 'dark matter'. I will then discuss experimental searches, both direct and indirect. Finally, I will offer prospects for the future.
\end{abstract}

(c)2002 Max-Planck-Gesellschaft and the authors. Further information on copyright is given at http://www.livingreviews.org/Info/Copyright/. For permission to reproduce the article please contact livrev@aei-potsdam.mpg.de. 


\section{Article Amendments}

On author request a Living Reviews article can be amended to include errata and small additions to ensure that the most accurate and up-to-date information possible is provided. For detailed documentation of amendments, please go to the article's online version at

http://www. livingreviews.org/Articles/Volume5/2002-4sumner/.

Owing to the fact that a Living Reviews article can evolve over time, we recommend to cite the article as follows:

Sumner, T. J.,

"Experimental Searches for Dark Matter",

Living Rev. Relativity, 5, (2002), 4. [Online Article]: cited on <date>, http://www.livingreviews.org/Articles/Volume5/2002-4sumner/.

The date in 'cited on <date>' then uniquely identifies the version of the article you are referring to. 


\section{Contents}

\begin{tabular}{lll}
\hline & Introduction & 4
\end{tabular}

2 The Evidence 5

2.1 Probes of Gravitational Wells . . . . . . . . . . . . . . . . . . 5

2.2 Toward a Standard Cosmological Model? . . . . . . . . . . . . 7

2.3 Discrepancies within the Standard Cosmological Model . . . . . . 10

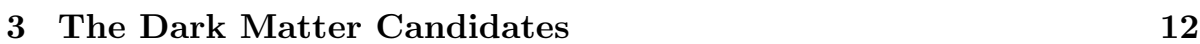

3.1 Baryonic Dark Matter . . . . . . . . . . . . . . . . . . . . . . . 12

3.2 Non-baryonic Dark Matter. . . . . . . . . . . . . . . . . . . . . . 14

3.3 Dark Matter in the Milky Way . . . . . . . . . . . . . . . . . . 15

\begin{tabular}{|lll}
4 & Non-Baryonic Dark Matter Detection & 17
\end{tabular}

4.1 Indirect Search Techniques f . . . . . . . . . . . . . . 17

4.2 Direct Search Techniques . . . . . . . . . . . . . . . . . . 18

5 Neutralino Detection Principles and Techniques 20

5.1 Expected Scattering Rates . . . . . . . . . . . . . . . . . 20

5.2 Expected Energy Deposit . . . . . . . . . . . . . . . . . . . . 22

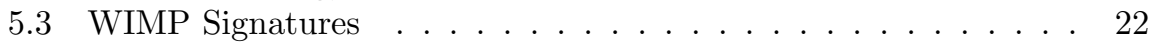

5.4 Neutralino Direct Detection Techniques . . . . . . . . . . . . 23

5.4 .1 Ionisation Detectors $\ldots \ldots \ldots \ldots . \ldots \ldots$

$5.4 .2 \quad$ Scintillation Detectors $\ldots \ldots \ldots \ldots \ldots$

5.4.3 Hybrid Detectors Using Both Scintillation and Ionisation 28

5.4 .4 Phonon Detectors - Bolometers . . . . . . . . . . . . 31

5.4.5 Hybrid Detectors Using Phonons and Scintillation/Ioni-

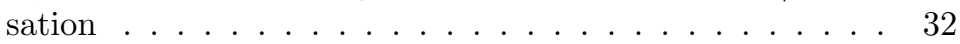

$5.4 .6 \quad$ Other Techniques. . . . . . . . . . . . . . . 32

6 Neutralino Project Overview and Limits 34

\begin{tabular}{|lll}
\hline 7 & Future Promises and Prospects & 40
\end{tabular}

\begin{tabular}{ll}
\hline References & 43
\end{tabular} 


\section{Introduction}

In the following section it will be seen that we do not yet understand what determines the gravitational potentials within the Universe on scales from galaxies upwards. One suggestion is that the Universe is full of material that does not emit electromagnetic radiation and which is thus hard to 'see'. There are a tremendous number of ideas on offer to resolve the dark matter dilemma. These ideas cover modifications to the laws of gravity, cold conventional matter, new particles, neutrinos with non-zero mass, and exotic objects, to name just a few. These will be discussed in more detail later. At the moment, there is no absolutely clear solution, although a number of the possibilities do lend themselves to experimental "searches'," which are being carried out around the world. Some of the experiments are designed to look for direct signatures, i.e. the physical interaction of a dark matter particle in a detector, while others are looking for indirect evidence, such as the neutrino or $\gamma$-ray flux due to dark matter particle annihilations. In addition, other types of experiments are looking for circumstantial evidence, such as the measurement of a non-zero neutrino mass in the right range, or confirmation of supersymmetry (SUSY). A number of the direct and indirect searches will be digging deeply into theoretically very interesting regions of parameter space over the next five years. Indeed there is, at this time, one experimental result from a direct search that has a signal consistent with the most optimistic SUSY predictions concerning neutralinos - which just might be proven correct. While it is true to say that most activity is currently targeted at what might be called the standard cosmological model, this model is not without its difficulties as a theory, and experimental evidence from dedicated dark matter searches could be crucial in finally clarifying the situation. Experimental searches probably are the only way to find out unambiguously what the Milky Way is made of.

The evidence is reviewed in section 2 , including subsection 2.2 on the standard cosmological model. The candidates are presented in section 3 . This leads to section 4 on direct and indirect detection requirements and techniques for non-baryonic dark matter. Most experiments are being carried out in the context of theoretical expectations for the neutralino, which most regard as the best motivated of the particle candidates. Section 5 looks in detail at the requirements for detection of the neutralino. A review of the current status of projects world-wide will be given in section 6 . Finally, in section 7 there is a discussion of the next logical steps for experimental searches for dark matter.

Living Reviews in Relativity (2002-4)

http://www.livingreviews.org 


\section{The Evidence}

\subsection{Probes of Gravitational Wells}

The existence of "dark matter" is inferred from astrophysical observations that probe gravitational potentials. The mass content required to provide the derived gravitational potential is then compared with the visible mass content. Several types of observation allow this to be done and in most cases the mismatch between the required mass and the observed mass is extreme. The following list summarises some of the evidence that has been accumulated:

- Studies of the dynamics of stars in the local disk environment gave rise to the first suggestion of 'missing matter' nearly 70 years ago [99, 100]. The kinetic energy associated with the motion of these stars normal to the plane of the Milky Way gives a measure of the restraining gravitational potential that binds them to the disk. Since the first work by Oort, a number of further studies have given conflicting results. However, even if present, this particular disk dark matter is not significant compared with the halo component.

- Rotation curves for a large number of spiral galaxies have now been reliably established and it is observed that the orbital velocities of objects (stars, globular clusters, gas clouds, etc.) tend to a constant value, independent of the radial position $r$, even for objects out toward, and even far beyond, the edge of the visible disks. This is quite inconsistent with the $1 / \sqrt{r}$ behaviour expected from Newtonian mechanics, assuming most mass is in the central part of the galaxies. According to Newtonian mechanics, the mass density within these galaxies is only declining as $\sim r^{-2}$, leading to a total mass that actually continues to increase proportional to $r$.

- Within the Local Group of galaxies, the Milky Way and Andromeda (M31) are approaching each other at a much faster pace than can be explained by gravitational attraction of the visible mass. To explain the approach velocity, and indeed the fact that these two galaxies are not still moving away from each other as part of the Hubble expansion, requires each to have masses that are consistent with those deduced from their rotation curves.

- Many clusters of galaxies show extended x-ray emission. This is usually attributed to a thin plasma of hot gas. On the assumption that the hot gas is gravitationally bound to the cluster and in equilibrium (i.e. we have a virial system), the gravitational potential energy can be inferred from the kinetic energy budget of the hot gas. The cluster mass determined in this way is much higher than that seen either visibly or in the gas itself.

- Gravitational lensing by clusters of galaxies causes images of more distant galaxies to be distorted and often split into multiple images. The gravitational mass of the lens (i.e. the cluster), and its distribution, can

Living Reviews in Relativity (2002-4)

http://www.livingreviews.org 
be recovered through detailed analysis of the image pattern surrounding the cluster. The lenses show a far more extended spatial extent than the visible cluster.

- Galaxy red-shift surveys have revealed large-scale galaxy-cluster streaming motions superimposed on the Hubble expansion. Attempts to explain this due to gravitational attraction resulting from the overall distribution of galaxy superclusters give the right direction of motion but need more than the observed visible masses in the superclusters to explain the speed of motion.

The next four items are not really at the same level of "simple" observational evidence as those above, as they require reliance on a more convoluted path to determine masses involved. However, the first three of these have received a great deal of effort and are now heavily used as a combined strong argument in favour of the existence of "dark matter", and indeed have resulted in a consensus view of "standard cosmology" over the past few years.

- The large scale structure (LSS) of the Universe can be studied using large surveys of distant galaxies, by measuring their spatial distribution and peculiar motions. There is an extensive industry in $N$-body simulations trying to explain the LSS and large-scale dynamics in terms of gravitational growth of small perturbations present in the early Universe. The only simulations that give reasonable agreement with observation are those that use a matter density somewhat higher than currently thought allowable in visible matter. Indeed, starting from the level of the COBE observations of the density fluctuations $\left(\sim 10^{-5}\right)$ at the time of recombination $(\mathrm{z}=1000)$, for gravitational instability to lead to galaxy formation on a reasonable timescale it seems necessary to invoke a significant dark matter component, which only interacts gravitationally.

- Type Ia supernovae can be used as standard candles to determine distances, independently of red-shift to high red-shift galaxies in which they occur. This allows the geometry of space-time to be studied at high redshift. The implications of the results will be discussed later, but consistent cosmological models seem to require a dark matter component.

- The COBE satellite gave us the first measurement of the amplitude of microwave background anisotropies at the time of recombination. It is these perturbations which subsequently grow through gravitational instabilities to form the large-scale structure seen today. COBE had a relatively poor angular resolution. Recently, new results have determined the angular power spectrum of the microwave background anisotropies at much finer angular scales, where enhancements are expected due to acoustic wave resonances in the early Universe. The position and amplitude of the enhancement depends on parameters of the early Universe. A clear first peak is seen in the data and its position favours a dark matter component. Even second and third peaks look to be emerging and the amplitudes and

Living Reviews in Relativity (2002-4)

http://www.livingreviews.org 
positions of these provide constraints on various cosmological parameters (this will be discussed in more detail in the following section).

- For those who believe in inflation, most surviving models naturally have a density equal to the critical density, which exceeds that possible in visible matter.

With such a large volume of evidence there can be no doubt that there is a real mystery to be unravelled here. Ideally, it would be satisfying if there were a single simple solution that explained all the above. This has proven elusive so far, but recently there has been some convergence on models that address the larger scale issues to do with the Universe as a whole, and this is discussed in the next subsection. The main aim is to establish a consensus opinion on the dark matter fraction, and more specifically the cold dark matter fraction, as this motivates most of the experimental searches for dark matter. In doing this we will see that a strong argument for a standard cosmology, with cold dark matter as one of its components, is beginning to become established. However, some issues clearly hint at aspects of the cosmology that have yet to be properly resolved, and some of these do have potentially serious implications for the cold dark matter component. These will be discussed in section 2.3 .

\subsection{Toward a Standard Cosmological Model?}

Within the context of a big bang creation scenario in which an inflationary phase is followed by expansion within the confines of General Relativity, there are at least 11 parameters that define a cosmological model in the post inflation era [36. These $11+$ parameters affect what happens in the first few minutes during which nucleosynthesis of the lightest elements occurs (BBN), how the geometry of space-time develops, and how structure forms through gravitational enhancement of primordial density inhomogeneities which come out of the inflationary phase. The density inhomogeneities will leave an imprint on the microwave background radiation that survives from the era of recombination, some 300,000 years after the big bang. Subsequently, they grow through gravitational instability to give rise to the structure we see in the distribution of visible objects in the Universe today. Hence, by bringing together observations that relate to BBN, space-time evolution, microwave background anisotropies, and large-scale structure, it is possible to define inter-related regions of the 11 parameter phase space that are consistent with all the information. Tegmark, Zalderriaga, and Hamilton [137 did this just after the first release of new CMB data from BOOMERANG [41] and MAXIMA [67. The parameter space used was

$$
\left(\tau, \Omega_{k}, \Omega_{\Lambda}, \omega_{\mathrm{d}}, \omega_{\mathrm{b}}, f_{\nu}, n_{\mathrm{s}}, n_{\mathrm{t}}, A_{\mathrm{s}}, r, b\right)
$$

where $\tau$ is the reionisation optical depth, $A_{\mathrm{s}}, n_{\mathrm{s}}, r, n_{\mathrm{t}}$ are the primodial amplitudes and tilts of the scalar and tensor inhomogeneities, $b$ is a bias factor relating rms galaxy fluctuations to the underlying rms matter fluctuations, $\Omega_{k}$

Living Reviews in Relativity (2002-4) http://www.livingreviews.org 
and $\Omega_{\Lambda}$ are the contributions to the overall density from curvature and the cosmological constant, $\omega_{\mathrm{d}}$ and $\omega_{\mathrm{b}}$ are the physical densities of dark matter (both hot and cold together) and baryonic matter, and finally $f_{\nu}$ is the fraction of dark matter in the form of hot dark matter. The primary observational data that they used were all available CMB data [54] and the recently released IRAS Point Source Catalogue Redshift Survey (PSCz) [113] from which they derived the large scale structure power spectrum. Simultaneous fits were then done to these data allowing all 11 parameters to vary [136.

\begin{tabular}{|c|c|}
\hline \hline $0.49 \leq \Omega_{\Lambda} \leq 0.74$ & $0.49 \leq \Omega_{\Lambda} \leq 0.76$ \\
$0.20 \leq \Omega_{\mathrm{m}} \leq 0.50$ & \\
$0.11 \leq h^{2} \Omega_{\mathrm{d}} \leq 0.17$ & $0.09 \leq h^{2} \Omega_{\mathrm{d}} \leq 0.17$ \\
$0.00 \leq h^{2} \Omega_{\mathrm{hdm}} \leq 0.12$ & \\
$0.10 \leq h^{2} \Omega_{\mathrm{cdm}} \leq 0.32$ & \\
$0.020 \leq h^{2} \Omega_{\mathrm{b}} \leq 0.037$ & $0.01 \leq h^{2} \Omega_{\mathrm{b}} \leq 0.03$ \\
\hline \hline
\end{tabular}

Table 1: Allowable ranges of values of density parameters within the standard cosmological model derived from the first release CMB data of BOOMERANG and MAXIMA (left-hand column, [137]) with corresponding values (where quoted) from the newest data sets (right-hand column, [143]). $h$ is the Hubble parameter and values of $0.74 \pm 0.08$ and $0.72 \pm 0.08$ were used in [137] and [143], respectively.

Table 2.2 shows the acceptable range of values for the key parameters that came out of those fits. In the table the matter density, $\Omega_{\mathrm{m}}=\Omega_{\mathrm{b}}+\Omega_{\mathrm{cdm}}+\Omega_{\mathrm{hdm}}$, includes both baryonic matter and dark matter, and moreover the dark matter can be classed either as "hot" or "cold" depending on whether it was relativistic or not in the early Universe. The total dark matter density is $\Omega_{\mathrm{d}}=\Omega_{\mathrm{hdm}}+$ $\Omega_{\mathrm{cdm}}$, and $h^{2} \Omega_{\mathrm{d}}=\omega_{\mathrm{d}}$. Of particular note for this review were that the cold dark matter density is non-zero and that the baryonic density has a range that just accomodates the constraints from BBN [27] at its lowest end, but with significantly better fits for higher values. The hot dark matter density can only be a minor component.

Very recently there have been significant new CMB data released from BOOMERANG [97, MAXIMA [64, DASI 77], and CBI 102]. These data have given better definition to the second and third peaks in the CMB power spectrum. Wang, Tegmark and Zaldarriaga [143] subsequently repeated the above analysis using a combination of these and previously available CMB data.

The two left-hand plots in the top row in Figure 1 are the most relevant for the dark matter. We see that the dark matter density is again non-zero, with a similar range of values as before, and that the fraction of dark matter as "hot dark matter" is less than $35 \%$, assuming no constraints on the hubble parameter, $h$. The allowable fraction of hot dark matter drops to only $20 \%$ if the

Living Reviews in Relativity (2002-4)

http://www.livingreviews.org 

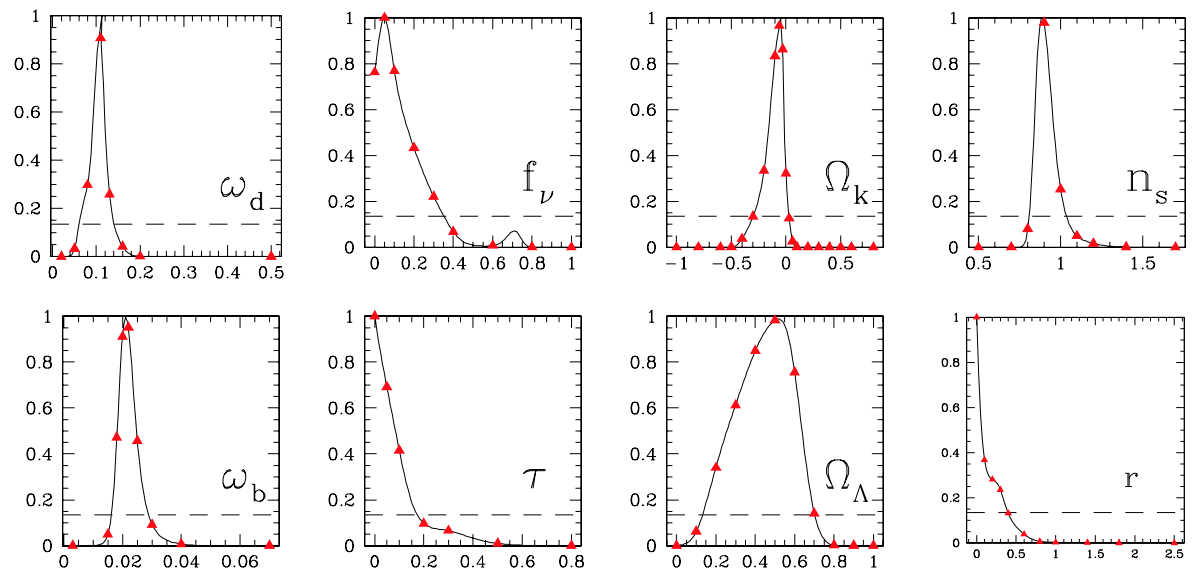

Figure 1: Allowable parameter spaces for $\Omega_{\Lambda}, \Omega_{k}, \omega_{b}, \omega_{d}, f_{\nu}, \tau$ and $r$. The figure is taken from [143] and the dashed lines mark the $95 \%$ confidence limits.

preferred hubble parameter value is imposed. The right-hand column in table 2.2 lists the quoted $95 \%$ confidence limits for comparison with the earlier analysis. The most striking difference is in the baryon density. While previously the allowable range of $\Omega_{b}$ was only just compatible with the upper limit derived from BBN [27, it now comfortably embraces it. This is illustrated in the left-hand panel in figure 2, which shows the combined constraints on the baryonic matter and dark matter densities. The white central region is the allowed parameter space when all constraints are applied, except for BBN of course. Relaxing the constraints by not using the $\mathrm{PSCz}$ data enlarges the allowed region to include the cyan coloured area. If, in addition, no assumptions are made about the value of the Hubble constant, then the green area also becomes allowed. If all constraints are accepted then figure 2 implies there is between 4.5 and 9 times as much dark matter in the Universe as there is baryonic matter.

Constraints on cosmological models can also be derived from the observations of high red-shift Type 1a supernovae [59. When combined with data from the CMB anisotropies, these limits give reasonable agreement with those cited earlier in table 2.2. A recent result from de Bernardis et al. [42] is shown in the right-hand panel in figure 2. This time what is shown are the joint constraints on $\Omega_{\lambda}$ and $\Omega_{m}$. The solid curves are the 1 to $3 \sigma$ combined likelihood contours and these can be compared with the values in the table. A somewhat weaker constraint on the Hubble constant was used.

Hence, from the above, there does indeed seem to be a cosmological model that can simultaneously satisfy all the observational evidence used. The ranges of values for the key parameters relevant to dark matter searches have been summarized in table 2.2. Rotation curves of galaxies can also be explained with this type of cosmological model. Numerous $N$-body simulations have been performed to verify whether structure formation occurs properly in a number

Living Reviews in Relativity (2002-4) http://www.livingreviews.org 

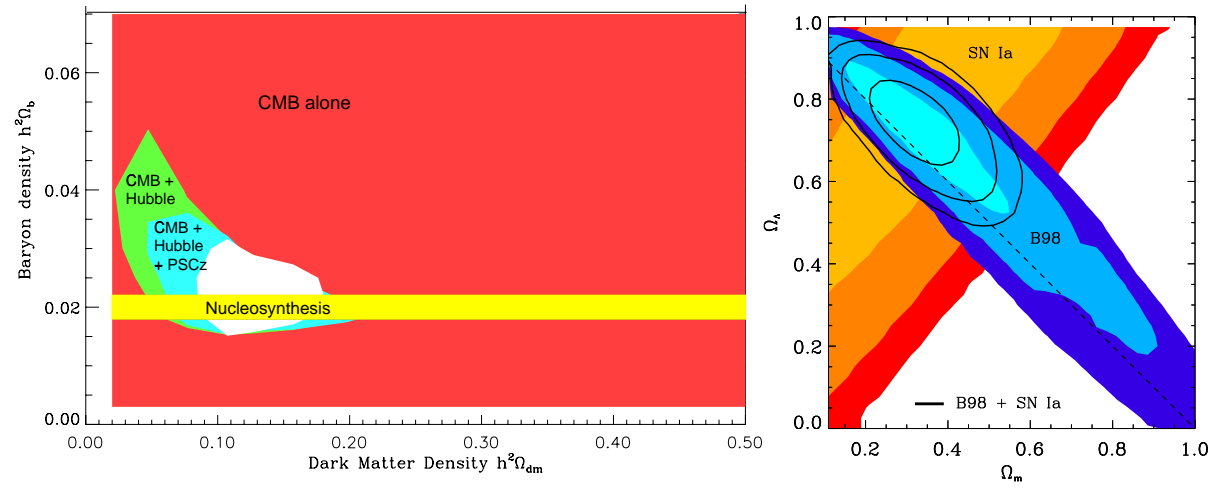

Figure 2: The left-hand panel from [143] shows the joint constraints on the baryonic matter and dark matter densities, together with the allowed band of baryonic density from BBN models. The right-hand panel from [42] shows the joint constraints on $\Omega_{\lambda}$ and $\Omega_{m}$ which result from combined use of CMB data and high-redshift supernova data.

of different types of models. Gawiser and Silk [53] reviewed the situation with regard to large-scale structure. Simulations of gravitational collapse on the scale of galaxies have resulted in universal rotation curves that match reasonably well those observed in a wide range of galaxies 94, 95.

From table 2.2 the main features of the emerging standard cosmology from the point of view of dark matter are:

- A $\Lambda$ CDM scenario where the dominant energy density is from $\Omega_{\Lambda}$.

- The matter density $\Omega_{\mathrm{m}}$ greatly exceeds that which can be present in a baryonic form, and hence there is a significant dark matter component.

- The dark matter density $\Omega_{\mathrm{d}}$ is mostly comprised of cold dark matter.

- $N$-body simulations suggest that galaxies are comprised of similar mixes of baryonic matter and dark matter as the universe as a whole.

The origin of $\Omega_{\Lambda}$ remains a topic of current debate, with a great deal of interest in quintessence [29, 9].

\subsection{Discrepancies within the Standard Cosmological Model}

Although the argument in section 2.2 is very persuasive and does present a formally consistent picture there are a number of concerns that continue to require attention.

Firstly, it can be seen from table 2.2 that, prior to the latest CMB data, the consistency between BBN and the CMB and LSS constraints was marginal. As a result of this a number of routes that allow for higher baryon density were

Living Reviews in Relativity (2002-4)

http://www.livingreviews.org 
explored. The most recent of these [48] invoked a degenerate BBN scenario in which additional light neutrinos (either sterile or degenerate) are allowed. Consistency with observed CMB anisotropies was obtained for $4 \leq N_{\nu} \leq 13$ with $0.25 \leq h^{2} \Omega_{\mathrm{b}} \leq 0.35$. Such a high baryon density would negate the need for dark matter. While it is comforting (to some) to see the new CMB data apparently removing this discrepancy, the data themselves are still not "highprecision" and some aspects of the data reduction remain uncertain [143].

A second issue has arisen from high resolution $N$-body simulations 96,57 , 90. These simulations seem to be showing a more peaked CDM enhancement toward galaxy centres than the previous work [94, 95] and more sub-structure in the CDM halos themselves 61. There is increasing evidence that indeed the predictions are incompatible with observational data 26, 112, 21, 43, 80. Possible ways of softening the central profile include allowing the dark matter to interact more readily, either with itself (self-interacting CDM [25]) or with baryonic matter. Although the $N$-body simulations themselves appear robust in general, in central regions where there are few "particles" there is the issue of resolution and convergence 91 .

Thirdly, it can be seen that the type Ia supernovae data are crucial in determining the value of $\Omega_{\Lambda}$. Central to this is the question of whether the optical light-curves can really be used as standard candles, or whether reddening is playing a role here, as quite small amounts of absorption could significantly affect the results. Use of infrared light curves may well be more reliable [83]. This suggestion has been countered recently by the observation of a very highredshift $(\mathrm{z} \sim 1.7)$ supernova which is actually brighter than expected, even in a "no-dust" scenario [108. Its increased brightness is shown to be consistent with an early deceleration phase of the Universe.

Finally, there is a class of model in which gravity itself is assumed to be modified [86, 85. A large number of effects attributed to dark matter have been addressed using modified gravity [82] with the most recent being an analysis of the latest $\mathrm{CMB}$ aniostropy data 81. In this latest work it is claimed that $\Omega_{\Lambda} \sim 1$ with $\Omega_{\mathrm{b}} \sim \Omega_{\mathrm{m}}$ (consistent with standard BBN) is the favoured model. However, the result rests heavily on the apparent absence of a second peak in the CMB data from BOOMERANG [41; MAXIMA-1 data [67] are not included. At the moment the totality of CMB data does not constrain the second peak sufficiently strongly to rule out a significant $\Omega_{\mathrm{cdm}}$ component.

Living Reviews in Relativity (2002-4)

http://www.livingreviews.org 


\section{The Dark Matter Candidates}

It was argued in section 2.2 that there is a clear need for dark matter. In addition, it seems the baryonic density must be very close to the maximum allowed by BBN. This last fact raises another interesting requirement in that the current best estimate places the baryonic density in the visible content of galaxies and cluster gas as $103 \Omega_{\mathrm{v}} \sim\left(2.2+0.6 h^{-1.5}\right) \times 10^{-3} \sim 0.003$. This is well below the range given in table 2.2 and implies that there is a lot more baryonic matter yet to be found. This missing baryonic matter is also generally referred to as "dark matter". In the following sections the possible forms of both the baryonic and non-baryonic dark matter will be reviewed. Table 2, adapted from Carr (1990) [30], summarizes some of the types of objects that have been suggested as dark matter.

\begin{tabular}{|lr|lr|}
\hline \hline \multicolumn{2}{|c|}{ Non-baryonic } & \multicolumn{2}{|c|}{ Baryonic } \\
\hline \hline Axions & $\left(10^{-5} \mathrm{eV}\right)$ & Snowballs & $?$ \\
Neutrinos & $(\sim 10 \mathrm{eV})$ & Brown dwarfs & $\left(\leq 0.08 M_{\odot}\right)$ \\
WIMPs & $\left(1-10^{3} \mathrm{GeV}\right)$ & M-dwarfs & $\left(0.1 M_{\odot}\right)$ \\
Monopoles & $\left(10^{16} \mathrm{GeV}\right)$ & White dwarfs & $\left(1 M_{\odot}\right)$ \\
Planck relics & $\left(10^{19} \mathrm{GeV}\right)$ & Neutron stars & $\left(2 M_{\odot}\right)$ \\
Primordial black holes & $\left(>10^{15} \mathrm{~g}\right)$ & Stellar black holes & $\left(\sim 10 M_{\odot}\right)$ \\
Quark nuggets & $\left(<10^{20} \mathrm{~g}\right)$ & Very Massive Objects & $\left(10^{2}-10^{5} M_{\odot}\right)$ \\
Shadow matter & $?$ & Super Massive Objects \\
Cosmic strings & $?$ & Cold diffuse gas \\
\hline \hline
\end{tabular}

Table 2: Possible dark matter candidates (adapted from [30]).

Figure 3 illustrates the scale-lengths on which the various dark matter candidates might be significant. The white areas are the allowed ones. The dark grey areas are strongly disallowed on theoretical grounds, whilst the lighter grey areas are unlikely but not rigorously excluded. In the next two sections a very brief discussion of the main baryonic and non-baryonic candidates is given. Carr (2000) 31] and Turner (1999) [138 have given more detailed reviews.

\subsection{Baryonic Dark Matter}

According to the standard model, baryonic dark matter is required to make up the difference between the visible matter density $\Omega_{\mathrm{v}}$ and the baryon density $\Omega_{\mathrm{b}}$ required by standard BBN models. Exactly where these baryons might be hiding depends on the nature of the objects being studied. For high redshifts $>3$, most of the baryons might still be in the form of an intergalactic medium still in the process of collapse 144, while recent data from a large sample of nearby x-ray emitting clusters of galaxies have shown that for these clusters

Living Reviews in Relativity (2002-4)

http://www.livingreviews.org 


\begin{tabular}{|c|c|c|c|c|}
\hline \multirow{2}{*}{\multicolumn{5}{|c|}{ SMO }} \\
\hline & & & & \\
\hline VMO & & & & \\
\hline $\mathrm{BH}$ & & & & \\
\hline NS & & & & \\
\hline WD & & & & \\
\hline $\mathrm{MD}$ & & & & \\
\hline $\mathrm{BD}$ & & & & \\
\hline Cloud & & & & \\
\hline $\mathrm{PBH}$ & & & & \\
\hline CDM & & & & \\
\hline HDM & & & & \\
\hline
\end{tabular}

Figure 3: Possible scale-lengths where different types of dark matter might be present, based on a similar representation which appeared in [30].

most of the baryon fraction $f$ is in the surrounding hot gas of the intracluster medium (ICM) 88. Indeed, if it is assumed that the cluster matter has the same fraction of baryonic matter as the universe as a whole, i.e. $f \simeq \Omega_{\mathrm{b}} / \Omega_{\mathrm{m}}$, then it seems that the ICM accounts for baryons up to the required BBN levels in these clusters. On the scale of individual galaxies the situation is much less clear [31. Rotation curves of galaxies imply the existence of dark-matter centrally clustered halos. $\Lambda$-CDM $N$-body simulations (see figure 4 ) suggest the halo composition should follow the underlying matter distribution of the universe but with some enhancement of the baryonic proportion through more efficient dissipative collapse.

This naturally leads to the conclusion that there is probably unseen baryonic matter in galaxies, but that it is unlikely to be sufficient to entirely explain the rotation curves. The brown dwarf (BD) candidate entry in figure 3 includes any compact object with masses below $0.08 M_{\odot}$. Many searches have been carried out looking for these MAssive Compact Halo Objects (MACHOs) using microlensing data. These are reviewed in [31. Although a number of candidate microlensing events have been seen, the apparent mass determinations for the lenses and their locations cast doubt on whether the lenses are indeed MACHOs in the halo of the Milky Way. The most recent estimates put the most likely MACHO contribution to the halo at 20\% [8, and the masses of these objects appear to be $\sim 0.5 M_{\odot}$. This suggests a population of white dwarfs and might indicate an early epoch of star formation in the Galactic halo. To explain all the dark matter with compact objects larger than brown dwarfs would have produced too many heavy elements during their evolution as stars prior to collapse and so these are still excluded as halo baryons in figure 3, at least as far as providing the bulk of the Galactic dark matter. However, above $\sim 10^{5} M_{\odot}$, super massive objects (SMOs) might collapse immediately to black holes. SMOs would still produce microlensing effects and would also give rise to dynamical effects, such as the heating of disk stars and the disruption of globular clus- 
ters [32]. Finally, it remains possible in principle that cold clouds with masses $\sim 10^{-3} M_{\odot}$ might provide some of the halo dark matter [104, 140, 141].

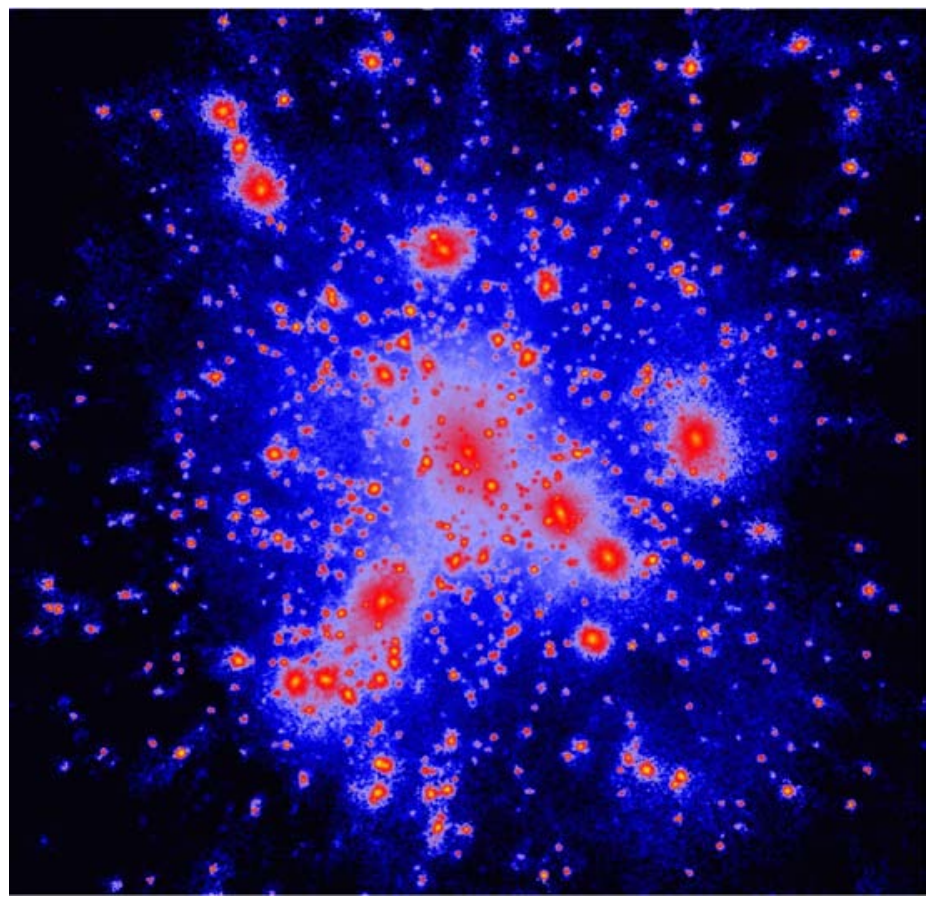

Figure 4: High-resolution N-body simulation of a galactic dark matter halo [90].

\subsection{Non-baryonic Dark Matter}

The non-baryonic candidates are classified as either hot or cold dark matter. Hot implies the matter was relativistic in the early universe. An example of hot dark matter is the neutrino. From table 2.2 it can be seen that the standard model still allows for a small HDM component. The neutrino would fulfill this if it had a non-zero mass, as suggested by recent experimental results from Super-Kamiokande [51, 71]. However, most of the dark matter will be cold dark matter (CDM). The best motivated of the various suggested candidates are two particles that were already invoked for other reasons. These are the axion and the lightest supersymmetric particle (neutralino), which is a member of the generic family of weakly interacting massive particles (WIMPs).

Axions are particles invoked to resolve the strong $\mathrm{CP}$ violation problem [74]. Without these particles, the level of $\mathrm{CP}$ violation seen in the $K_{0}$ decay would give rise to a neutron electric dipole moment in excess of the limits already established. The allowable mass range for the axion is constrained by astrophysical arguments to $10^{-3}$ to $10^{-6} \mathrm{eV} / \mathrm{c}^{2}$.

Living Reviews in Relativity (2002-4)

http://www.livingreviews.org 
WIMPs are naturally predicted in supersymmetry theories in which a higher level symmetry is obtained in the particle families by introducing new particles to match each of the known particles. In the so-called minimal supersymmetry models (MSSM), the lightest supersymmetric particle (LSP) is likely to be a neutralino $\chi$, which is a mixture of two neutral gauginos and two neutral higgsinos [79]:

$$
\chi=\chi_{1}^{0}=f_{11} W^{3}+f_{12} B+f_{13} H_{1}+f_{14} H_{2} .
$$

There are numerous parameters required to specify a particular MSSM configuration. It turns out that there is a wide range of parameter space in which the production and annihilation rates in the early universe are such that $\Omega_{\mathrm{LSP}} \simeq$ $\Omega_{\mathrm{cdm}}$ [45. The mass range for neutralinos is $46 \mathrm{GeV} / c^{2} \leq m_{\chi} \leq 2000 \mathrm{GeV} / c^{2}$, where the lower limit comes from accelerator data from LEP [66].

\subsection{Dark Matter in the Milky Way}

Experimental searches for dark matter invariably are trying to detect cold dark matter within our own galaxy. Thus, it is useful to review at this stage the current thoughts about the distribution of cold dark matter within the Milky Way and, for terrestrial based experiments, the likely cold dark matter presence near Earth. Figure 5 shows both the observational data on the rotation curve [50. and a recent determination of various mass components [72].
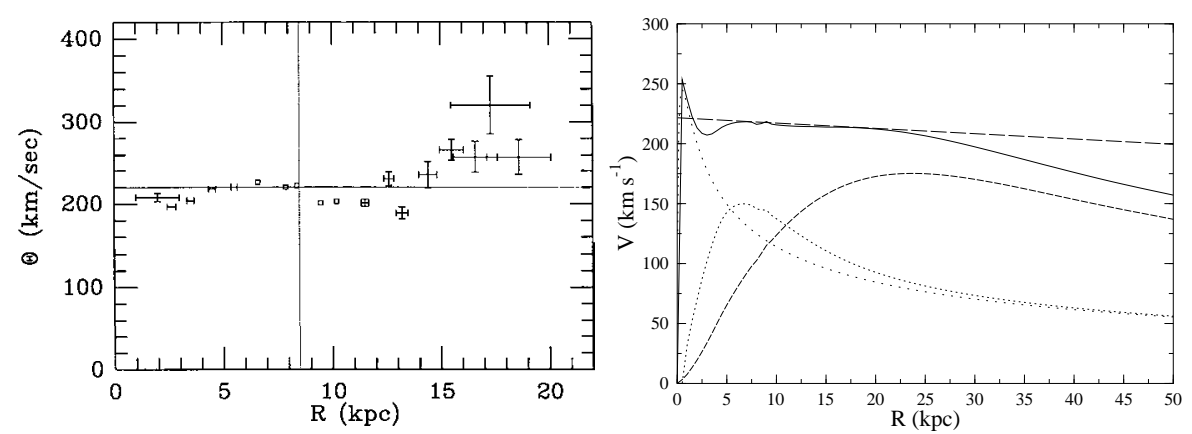

Figure 5: The rotation curve of the Milky Way. In the left-hand panel are the measured rotation speeds given by the average values from a number of measurements on different objects [50]. The right hand panel shows the various mass components that combine together to reproduce the observed curve between 5 and $25 \mathrm{kpc}$ [72]. The dotted lines are the bulge and disk contributions, and the short-dashed curve is the dark matter contribution. The solid curve shows the combined effect of all three, and this is compared to the long-dashed curve which approximates the measured data in the left-hand panel below $25 \mathrm{kpc}$.

At the position of the Sun, $7.5-8 \mathrm{kpc}$, it can be seen that the contributions to the enclosed mass from the bulge, the disk, and the dark matter halo are 
comparable. In these types of studies, the dark matter halo is assumed to be in a quasi-spherically symmetric distribution in virialised equilibrium. The halo is usually taken to be non-rotating and the local density comes out as $\sim 0.3 \mathrm{GeV} / \mathrm{cm}^{3}$. The velocity distribution of the DM particles is assumed to be Maxwellian with an upper cut-off at the Galactic escape velocity. Most calculations of event rates and energy deposits in detectors are done assuming this straight forward type of DM halo [78]. Possible modifications to this simple DM geometry include:

- A modified radial CDM density profile giving a much lower CDM local density. This has been implied recently by analysis of microlensing events towards the Galactic bulge. A much larger number of events have been seen than expected and this suggests an unseen stellar population within the solar radius that can apparently account for the local rotation speeds without the need for dark matter [56].

- Gravitational clustering of CDM particles at the centres of massive objects [124, such as the Sun, the Earth, or the galactic centre.

- CDM halos with non-zero angular momentum.

- Clumpy CDM galaxy halos [57, 90, 92.

- Non-equilibrium situations with on-going CDM infall into the Galaxy [116, 115.

- Non-equilibrium situations with on-going CDM infall into the local cluster/supercluster.

- CDM scattered into stable orbits around the Sun [40].

Living Reviews in Relativity (2002-4)

http://www.livingreviews.org 


\section{Non-Baryonic Dark Matter Detection}

There are a number of previous reviews on general techniques used for dark matter searches [105, 119, 131. Two basic methods can be used, either direct or indirect. Direct searches depend on dark matter particles actually passing through detectors and physically interacting with them. Indirect searches look for secondary products produced when dark matter particles annihilate each other elsewhere. Direct searches can, in principle, be used to look for neutrinos, axions and WIMPs, whereas only WIMPs are accessible indirectly.

\subsection{Indirect Search Techniques}

WIMP particle-antiparticle annihilations can produce neutrinos, $\gamma$-rays, antiprotons, and positrons. Experiments have been proposed to detect all of these [49. For neutrinos and $\gamma$-rays the signal rates expected depend on the WIMP-antiWIMP densities. SUSY neutralinos (the WIMPs on which most attention is fixed) are their own antiparticles and the annihilation process can be represented as $\chi \bar{\chi} \rightarrow f \bar{f}$ or $\rightarrow W^{-} W^{+}$or $\rightarrow Z Z$, where $f=\nu, \tau, c, b, t$ with $c, b, t$ being quarks.

Neutrino signal rates can be enhanced by the trapping of WIMPs in massive bodies, such as the Sun, Earth, or Galactic centre; the WIMP density builds up until the annihilation rate equals the capture rate. For the Sun this equilibrium situation has already been reached. For Earth this may not yet be the case and annihilation fluxes may be only $10 \%$ of that expected in equilibrum. The capture rate will depend on the scattering rates for WIMPs on the various nuclear species in the body and the energy transfer per scatter. The scattering rate on a particular species will depend on the abundance of the species and the cross-section. The scattering cross-sections are usually calculated 49] within MSSM constraints, abundances depend on which body the WIMPs are being trapped in, and energy transfer per collision normally assumes elastic scattering with the WIMPs starting out with a typical virial speed of $\sim 10^{-3} c$ for particles bound to the Galaxy. Once capture rates, and hence annihilation rates, have been derived, the neutrino flux is calculated from the branching ratios for WIMP annihilations going into neutrinos. Neutrino products are typically in the GeV energy range and are hence accessible to existing solar neutrino experiments. However, for contained events (ones in which the muons produced by the neutrinos are stopped in the detector) the predicted rates $R_{\mathrm{c}}$ are a few events for kiloton of detector per year, while traversing signals (muons produced in surrounding rocks and passing through the detector) occur at a rate $R_{\mathrm{t}} \sim 0.1 R_{\mathrm{c}} E_{\nu}\left(A / 10^{6}\right)^{-1} \mathrm{yr}^{-1} . A$ is the detector area in $\mathrm{cm}^{2}$. Results from this type of experiment first appeared in the mid-1980s [46].

Early studies of $\gamma$-ray signatures from WIMP annihilation predicted both continuum emission from $\pi_{0}$ products, and line features from $\chi \bar{\chi} \rightarrow \gamma Z$ and direct WIMP annihilation into photons $\chi \bar{\chi} \rightarrow \gamma \gamma$ [129, 130, 49]. Continuum emission fluxes were predicted to be about two orders of magnitude lower than the diffuse galactic background. However, some enhancement would be expected

Living Reviews in Relativity (2002-4)

http://www.livingreviews.org 
in the direction of the galactic centre. Line emission features should be much easier to see above the background as long as good energy $(\Delta E / E \sim 1 \%)$ is available.

Antiproton fluxes from WIMP annihilation were expected to produce measurable enhancements above typical background fluxes in the low-energy antiproton spectrum $(<1 \mathrm{GeV})$, which would be accessible to space instruments such as AMS 4. However, it is now thought that there will be additional background fluxes that will make this type of measurement difficult.

Positron features around 50-100 GeV are expected from neutralino annihilations. These may be visible as bumps in the otherwise smooth background spectrum due to cosmic-ray interactions with interstellar gas. Signals are expected to be much below the background levels, and long-duration space missions will be needed to collect sufficient statistics to observe the positrons [49].

\subsection{Direct Search Techniques}

Although there have been a number of suggestions for experiments to detect neutrinos [119, 123] (residual primordial hot dark matter now gravitationally bound to the galaxy), none can yet achieve sufficient sensitivity.

Axions, on the other hand, are amenable to direct detection [109], although it is challenging to fully explore the whole of the theoretically available parameter space. Among particles proposed to solve the $\mathrm{CP}$ violation problem, the axion comes in two varieties, which have fairly well defined properties [75]. Axions can be converted completely into photons in what is essentially a two-photon interaction. In experiments to detect galactic dark-matter axions the second photon is provided by an intense ambient electromagnetic field. The photon created has an energy equal to the total energy of the axion (rest mass plus kinetic energy). As noted earlier, the dark matter energy density at the position of the Earth is about $0.3 \mathrm{GeV} / \mathrm{cm}^{3}$. The preferred mass range for the axion is between $10^{-6}$ and $10^{-3} \mathrm{eV} / \mathrm{c}^{2}$, although there is a second window between 2 and $5 \mathrm{GeV} / c^{2}\left[139\right.$. The lower limit of the preferred mass range keeps $\Omega_{\mathrm{m}} \leq 1$, while the upper limit prevents excessive energy-loss mechanisms in stars and supernovae due to axion production and loss. If the galactic dark matter is axions, then their local density is between $3 \times 10^{11}$ and $3 \times 10^{13} \mathrm{~cm}^{-3}$. With a virial velocity distribution $\left(\sim 10^{-3} c\right)$, the flux through a terrestrial detector is enormous, but unfortunately the two-photon conversion process is very weak. In an ambient 6 Tesla field each axion has a conversion probability around $10^{-17}$ per second, and the photon produced has an energy in the microwave region $(2-200 \mathrm{GHz})$. Such an experiment requires a tuned high-Q cavity, tunable over the projected axion mass/energy range, with a sensitivity of around $10^{-23} \mathrm{~W}$. Two early experiments of this type [93, 84, 63] have been followed by a number of second generation instruments [109], and the preferred axion mass window has been closed over a very small range at its lowest end $\left(2.9 \times 10^{-6}\right.$ to $3.3 \times$ $10^{-6} \mathrm{eV} / \mathrm{c}^{2}$ ) at the $90 \%$ confidence level for KSVZ axions 62. A variant on the tuned cavity technique is to incorporate Rydberg atoms into the cavity where the $|n\rangle$ to $\left|n^{\prime}\right\rangle$ transition is also resonant with the cavity [145]. In addition

Living Reviews in Relativity (2002-4)

http://www.livingreviews.org 
to the direct dark matter axion searches, there are a number of experiments looking for evidence of axion existence, such as axion telescopes pointed at the Sun [87] and torsion balance instruments looking for short-range weak force spin-coupling interactions of the type mediated by the axion [122, 98, 114, 134]. These have yet to achieve sufficient sensitivity.

Neutralinos have received by far the most attention and there are an enormous range of techniques being used to search for these particles [119, 132, 6]. The basic questions that need to be addressed to assess the feasibility of detection of WIMPs in the halo of our Galaxy are:

- How often will scattering events occur?

- How much energy will they deposit?

- How easy will it be to separate any real signal from background and to convincingly prove that a signal has been seen, i.e. what characteristic signatures are expected?

Each of these three issues are dealt with in some detail for the neutralino of the MSSM in the following sections.

Living Reviews in Relativity (2002-4)

http://www.livingreviews.org 


\section{Neutralino Detection Principles and Techni- ques}

\subsection{Expected Scattering Rates}

The scattering rate per unit detector volume $R_{t}$ depends on the local density of dark matter particles $N_{\mathrm{w}}$; their velocity distribution relative to a detector in a terrestrial laboratory $n_{\mathrm{w}}(v)$, and the velocity dependent scattering cross-section $\sigma_{\mathrm{v}}$, via the usual equation

$$
R_{t}=\int_{0}^{\infty} n_{\mathrm{w}}(v) n_{t} \sigma_{t}(v) v d v
$$

where $n_{t}$ is the number density of nuclei of species $t$ in the detector. The local number density of WIMP particles is $N_{\mathrm{w}}=\int_{0}^{\infty} n_{\mathrm{w}}(v) d v=\rho_{\mathrm{cdm}} / m_{\mathrm{w}}$, where $m_{\mathrm{w}}$ is the WIMP mass and $\rho_{\text {cdm }}$ is the assumed local cold dark matter density. The WIMP velocity distribution and the cross-section both have a wide range of uncertainty, which makes accurate predictions impossible. The preferred range for $m_{\mathrm{w}}$ in the context of the lightest stable neutralino within minimal MSSM is 20 to $200 \mathrm{GeV} / c^{2}$ [110, 111], and Han and Hempfling [66] quote a lower mass limit from LEP data as $\sim 46 \mathrm{GeV} / c^{2}$. In the simplest models the dark matter density distribution in the halo of the Galaxy is taken to be a spherical $1 / r^{2}$ (at least for large $r$ ) distribution with a local density, at the position of the solar system, of $\sim 0.3 \mathrm{GeV} / \mathrm{cm}^{3}$. The velocity distribution is taken to be a Maxwellian, consistent with a virialised system but truncated above the Galactic escape velocity. Models involving non-spherical density distributions [73, rotating halos [44, 73, and/or non-virial velocity distributions, such as Galactic in-fall components with cusps [116] or bound Solar-System Earth-crossing components [40, can individually give factor-of-two differences in the predicted scattering rates. The WIMP velocity distribution as seen by a terrestrial detector has a bias imposed by the Earth's velocity through the halo and its spin. This produces a temporal modulation of the apparent WIMP velocity distribution, which results in an annual modulation of the WIMP scattering rate and recoil spectrum, and daily and annual modulations in the directional distributions.

The scattering cross-section itself has a very wide range of possible values [45]. Different neutralino models, within MSSM or SUGRA (supergravity), exhibit an enormous range of interaction strengths that can be pure axial in nature (coupling only to nuclei with non-zero spin), pure coherent (coupling to all nucleons), or any combination of the two. Figure 6 shows the allowed

range of parameter space for the scattering cross-sections. The plot [79] has been produced using output from the DarkSusy [58] code, using up to 65 free parameters. Even in this plot some 'reasonable' assumptions have been made in allowing the parameters to vary; Ellis [45] relaxes some of these and, not surprisingly, finds a wider range of resulting cross-sections. The cross-sections are normalised to one nucleon; to calculate the total cross-section for a target nucleus with $N$ neutrons and nuclear spin $J$ requires a scaling as $\sim(N / 2)^{2}$ for

Living Reviews in Relativity (2002-4)

http://www.livingreviews .org 
the coherent spin-independent part of the cross-section and $\lambda_{\mathrm{s}}^{2} J(J+1)$ for the spin-dependent part. The value of $\lambda_{\mathrm{s}}$ depends on the target material 60].

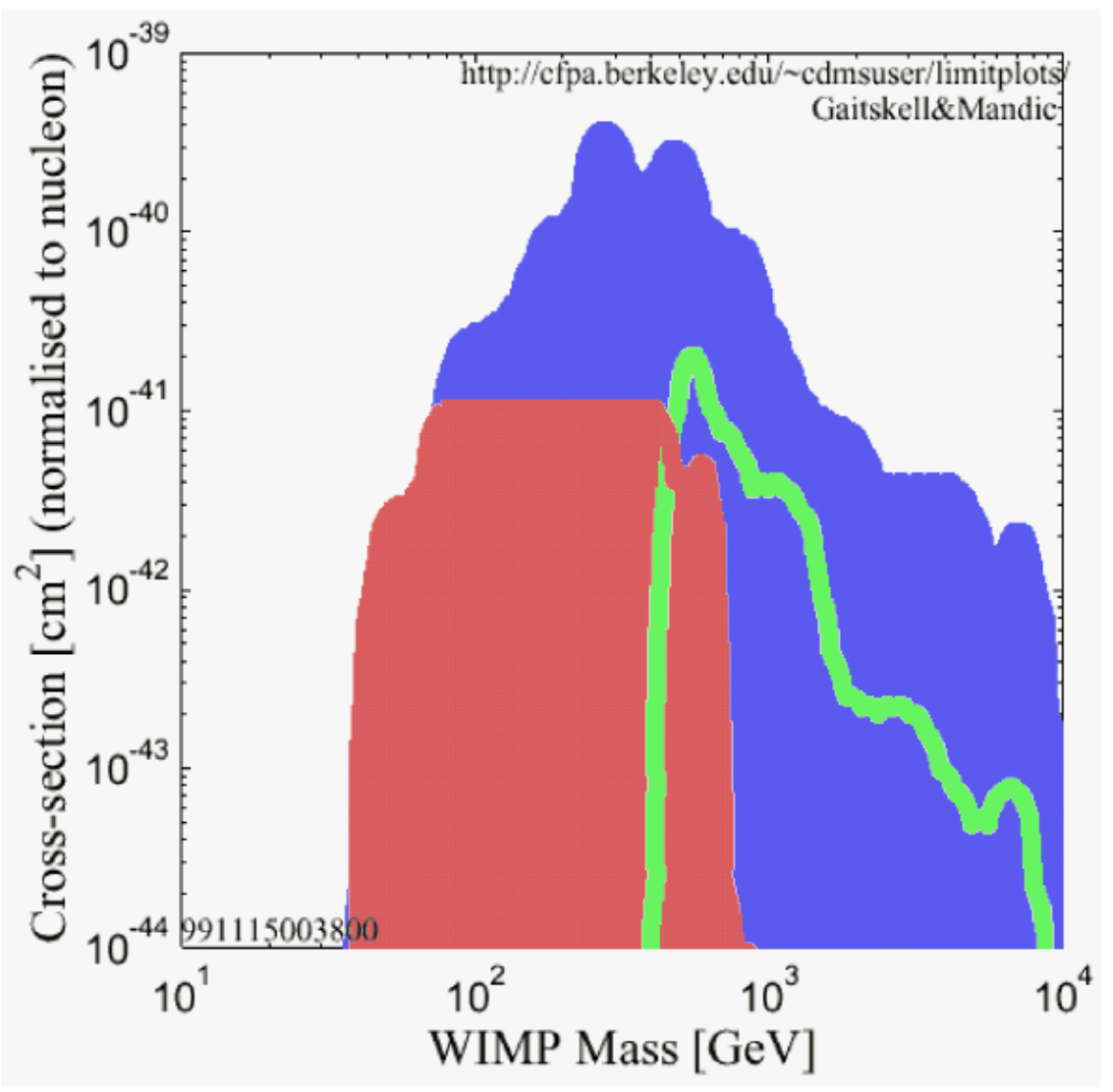

Figure 6: Total neutralino elastic scattering cross-section normalised to one nucleon for a range of neutralino models within MSSM and mSUGRA, taken from [79]. The pink area corresponds to a neutralino in a dominantly bino state, the green bounded area is dominantly higgsino. The cross-section includes both spin-independent and spin-dependent contributions, and in general the spindependent part is likely to be larger.

Form factor effects, which arise due to the finite size of the nucleus, are significant for the heavier target nuclei, are different for axial and coherent scattering, and again have uncertainties [47, 107]. Predicted event rates typically range from $10^{-4}$ to 10 events/day $/ \mathrm{kg}$. To achieve sensitivity to such rare events requires low-background instruments operating in well shielded underground environments.

Living Reviews in Relativity (2002-4) http://www.livingreviews.org 


\subsection{Expected Energy Deposit}

The predicted differential observable recoil spectrum, assuming a standard virial Maxwellian velocity distribution, is of the form

$$
\left.\frac{d R}{d E}\right|_{\text {obs }} \propto \frac{R_{0}}{f E_{0} r} e^{-E /\left(f E_{0} r\right)} F^{2}(E),
$$

where $f$ is the quenching factor which reflects the relative efficiency with which the nuclear recoil energy is recorded in the signal channel compared to a $\gamma$ ray of the same energy. $E_{0}$ is the characteristic energy for the virialized WIMP population, $F$ is the form factor referred to previously, and $r$ is a kinematic factor dependent on the relative masses of the WIMP and target nuclei. Somewhat more complex formulae result when modified velocity distributions are used (e.g. incorporating a Galactic cut-off), or the rate is convolved through the instrument response. A falling recoil spectrum with a maximum recoil energy $\sim 100 \mathrm{keV}$ is expected. For larger target nuclei, such as iodine, the form factor further suppresses the recoil spectrum above $50 \mathrm{keV}$.

Inelastic WIMP scattering (producing excited nuclear states) can also occur. $\gamma$-rays emitted from nuclear relaxation can be self-absorbed in the detector producing characteristic spectral lines. Experiments of this sort have been performed [15, 52, 18]; the cross-sections are much lower than the elastic ones and the technique will not be discussed further.

\subsection{WIMP Signatures}

A crucial quality of instruments and techniques is their ability to look for the signatures expected from WIMP scattering interactions. There are, in fact, a number of specific characteristics to be looked for, including:

- A characteristic (but featureless) recoil spectrum (following equation (4)) that depends on target nuclear mass and spin.

- Events distributed uniformly throughout the detector.

- An expected annual modulation in both the event rate and the recoil spectrum (since a component of the Earth's orbital velocity around the Sun effectively adds to and subtracts from the Solar System orbital velocity around the Galactic Centre).

- An expected daily modulation in the scattering rate due to WIMP scattering by the Earth's effectively shadowing the incident flux 68.

- A directional modulation in detector co-ordinates on daily and yearly bases for detectors locked to the Earth's surface.

- Site-independent WIMP parameters provided by the WIMP signal.

Living Reviews in Relativity (2002-4)

http://www.livingreviews.org 
- Characteristic "properties" for each scattering event, where the instruments being used have an intrinsically different response to WIMP nuclear recoil events as opposed to other backgrounds. For example:

- WIMP scattering should be single-site, whereas $\gamma$-ray and neutron background can be multi-site. Anti-coincidence veto systems are often used by ionization/scintillation detectors to provide multi-site signals.

- Nuclear recoil events characteristic of WIMP scattering produce different linear ionisation densities, which can result in different production ratios and different rates at which subsequent secondary processes occur. This can produce different pulse shapes for nuclear recoils as opposed to x-ray and $\gamma$-ray background events. This technique is commonly used in scintillation type experiments.

- Similarly, the different linear ionisation densities can affect the relative efficiency with which energy propagates into different signal channels. For example, the pulse height ratios between scintillation and ionisation signals are often used.

- Similarly, higher linear ionization density implies a much shorter range for the nucleus before it loses all its energy. Imaging scintillation instruments or time projection chambers can make use of this.

In the next section the various techniques on offer will be reviewed in order of increasing complexity in their ability to exploit specific WIMP signatures.

From the above dicussion it can be seen that an ideal detector would have:

- Energy threshold $<1 \mathrm{keV}$.

- Good energy resolution, to be able to see subtle modulations in the recoil spectrum.

- High ability to discriminate between nuclear recoil events and background events.

- Low-background construction and site for operation.

- High target mass to ensure a sufficiently high WIMP count rate.

- Stable operation over a number of years.

\subsection{Neutralino Direct Detection Techniques}

\subsubsection{Ionisation Detectors}

The first instruments to be used for WIMP searches were solid state germanium ionisation type detectors [28]. These recorded high-resolution background

Living Reviews in Relativity (2002-4)

http://www.livingreviews.org 

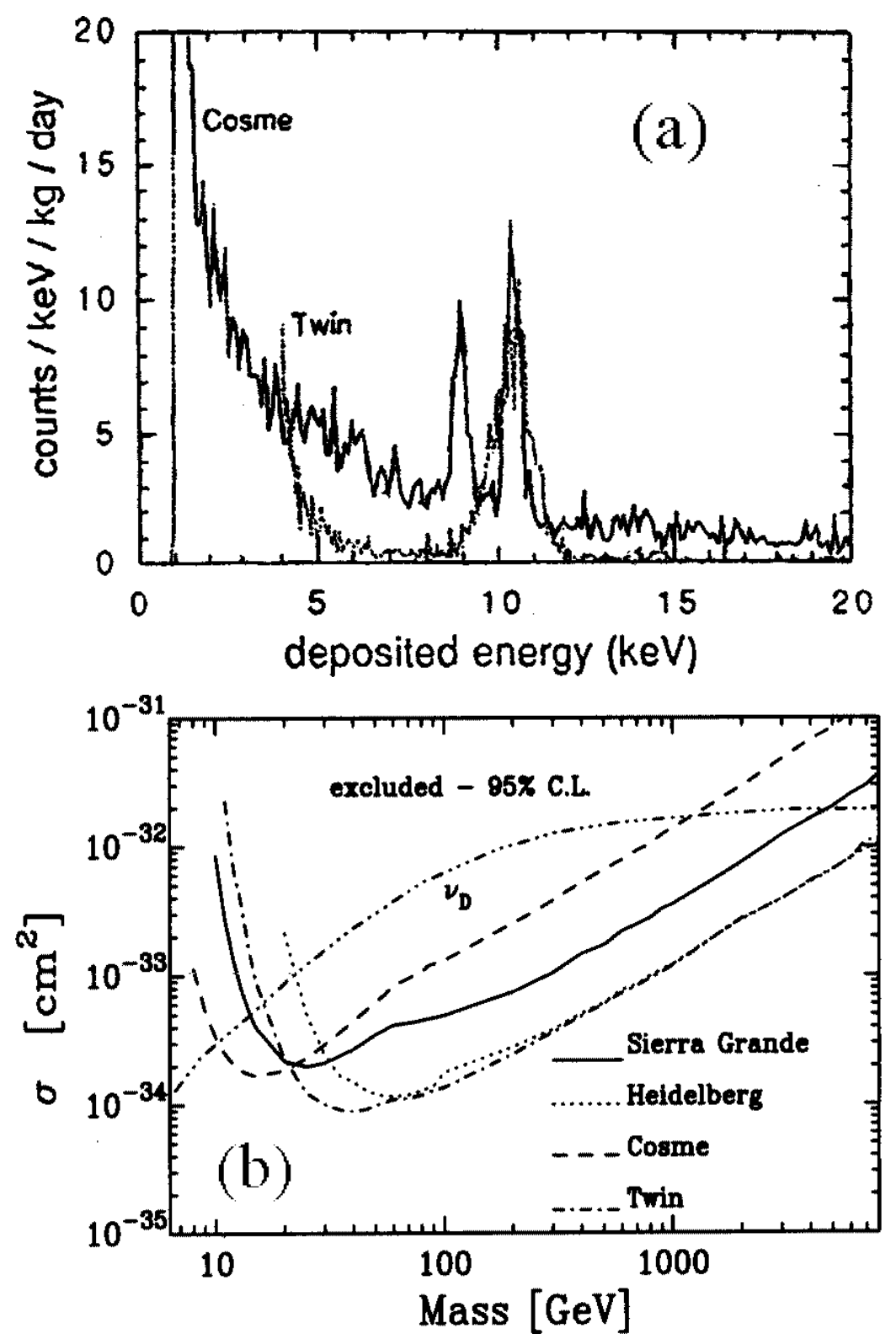

Figure 7: Background energy spectra for two Ge detectors of the PNL/USC/Zaragoza group taken from [28] (a - upper panel). Coherent crosssection upper limits from Ge detectors taken from [1] (b - lower panel). 
energy spectra, which were then compared to the expected WIMP recoil spectra to establish upper limits on interaction cross-sections (assuming the Galactic dark matter was indeed made of WIMPs in a straightforward spherical virialised distribution). Figure 7 shows examples of such spectra 28 and the coherent limits [1] obtained from a number of experiments of this type. The background spectra can be coarsely characterised by two parameters, which are the threshold and the count rate just below threshold. These approximately determine how low in WIMP mass the instrument sensitivity extends and how low a crosssection limit can be set respectively. This can be seen by comparing the Cosme and Twin curves in the two panels of figure 7 . The difference between the Cosme and Twin background spectra is due to the use of freshly mined germanium in the production of Twin, which consequently does not show the cosmogenically activated line just below $9 \mathrm{keV}$. An alternative way of achieving the same suppression of the cosmogenic lines is to use enriched germanium as done by the Heidelberg/Moscow experiment [13. The Sierra Grande curve in figure 7 is from a long exposure germanium experiment in which a search for both daily and annual modulation has been performed [1, 2, and the results from the daily modulation search are shown in figure 8 . No significant signals are seen. An example of an annual modulation search is shown in the right-hand panel of the figure. This is actually from a scintillator experiment [16] and this will be discussed later. The next advance expected from germanium detectors of this type will be from the Heidelberg group [12] who are developing a high-purity natural germanium crystal surrounded by an active veto that also uses natural germanium. This will exploit the fact that any WIMP scattering events will be single-site due to the very low scattering cross-section, while most other background events will be multi-site (e.g. multiple elastic neutron scattering or multiple compton scattering for $\gamma$-rays).

\subsubsection{Scintillation Detectors}

The next most common type of instrument in use is the scintillator, either as a solid crystal or as a liquid. NaI has been used most effectively to date. The predominantly non-zero nuclear spin of both natural $\mathrm{Na}$ and I make these detectors much more sensitive to axial couplings than natural germanium. While their energy thresholds and resolutions tend to be significantly worse than for germanium detectors, scintillators offer an additional discriminatory power in that the individual scintillation signals from nuclear recoil type WIMP interactions are expected to show a different time profile from that of the background. This has been studied in some detail by various authors [11, 55]. Figure 9 shows measured comparative differential pulse shapes from the UKDMC NaI group [106, 118. Figure 10 compares $\gamma$-ray and neutron induced nuclear recoil calibration time constant distributions (using simple single exponential fits to each pulse) with the background obtained from their working experiment. The closer statistical match of the measured background to the $\gamma$-ray distribution allows the upper limits to be reduced accordingly.

Another advantage of some scintillators over germanium is that it is much

Living Reviews in Relativity (2002-4)

http://www.livingreviews.org 

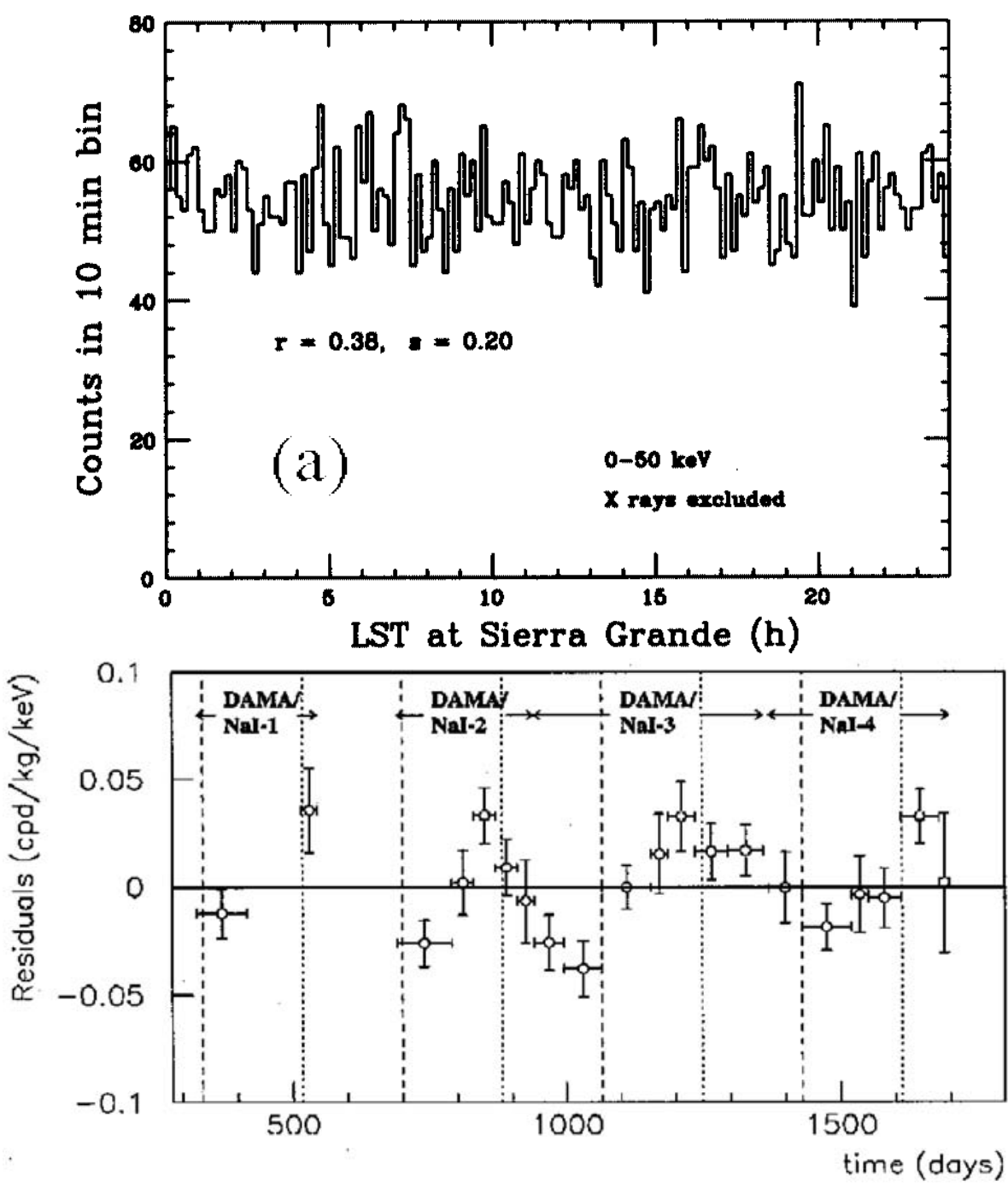

Figure 8: Background rate from 428.1 days of data binned in 10-minute intervals and folded to look for daily modulation [1] (a - upper panel). Results of an annual modulation search using 4 years of data from the DAMA experiment [16] (lower panel). 


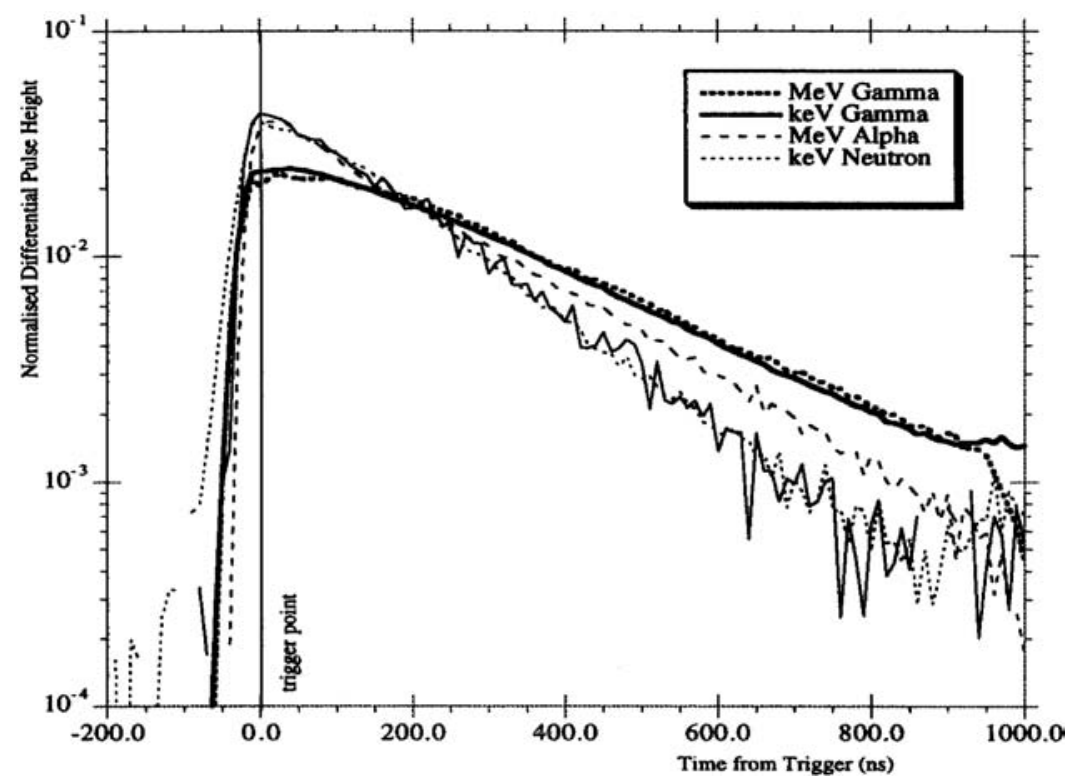

Figure 9: Differential pulse shapes from NaI for various radiation types. There is a clear difference between the functional form for high $d E / d x$ interactions, such as nuclear recoil and alpha tracks, and $\gamma$-ray induced electron tracks.

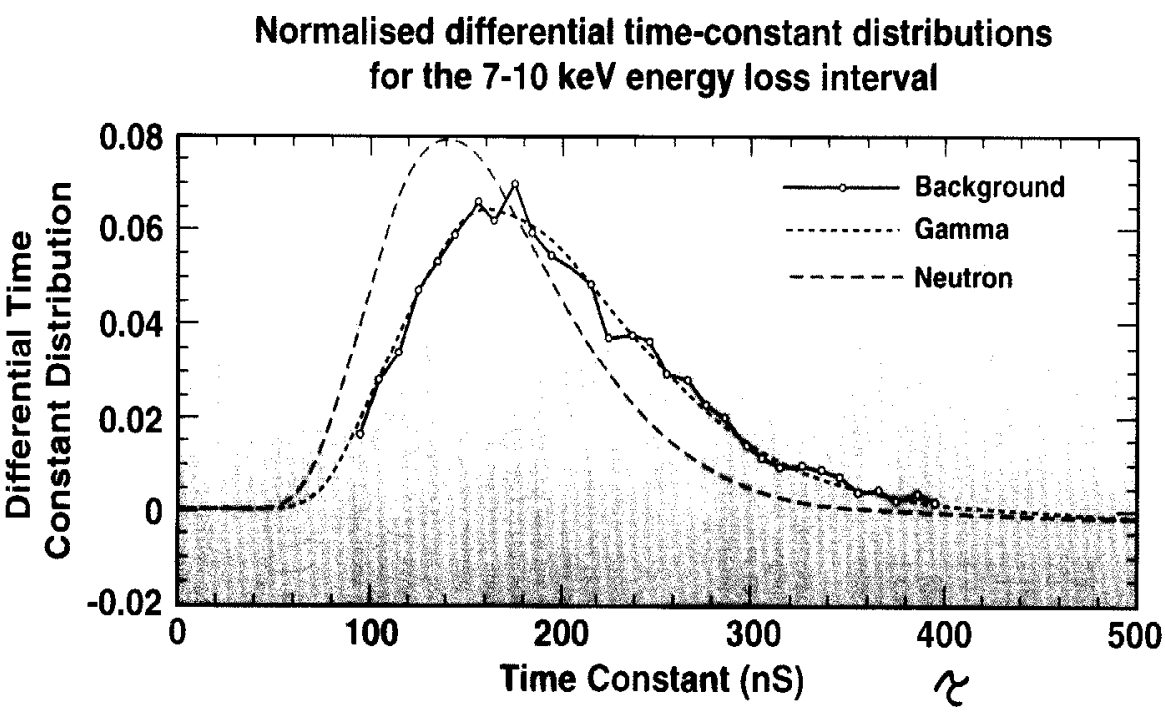

Figure 10: Differential time constant distributions from the UK NaI experiment [106, 118] showing the measured background (solid line + data points), and neutron and $\gamma$-ray calibration distributions.

Living Reviews in Relativity (2002-4) http://www.livingreviews.org 
easier to make large mass detectors out of them. This increases the event rate and makes it feasible to look for any annual modulation signals, assuming experiment systematics can be kept under control. This is the approach of the DAMA group [19, 17, 20, who currently have some of the lowest axial and coherent limits, and who have claimed a positive annual modulation result [16] (see right-hand panel in figure 8 and later discussion). Other 'simple' scintillators that are in use include $\mathrm{CaF}_{2}$ and liquid xenon. Various other effects in scintillators are also being studied as a means to provide additional discrimination against non-nuclear recoil backgrounds. These include using the ratio of visible to UV light emitted by cooled undoped $\mathrm{NaI}$ 127, looking for directional nuclear recoil effects in stilbene 128, and using pulse-shape analysis from a mixed scintillator system(with fine grains of $\mathrm{CaF}_{2}$ in an organic liquid scintillator) to take advantage of the recoil range difference between electrons and nuclei [126].

\subsubsection{Hybrid Detectors Using Both Scintillation and Ionisation}

All the previous techniques make use of only one diagnostic signal channel, i.e. pulse shape discrimination, annual modulation, pulse height ratio, or directional dependence. A technique that makes use of two distinct signal channels using a two-phase (liquid/gas) xenon detector has been demonstrated and is under development by the UKDMC group. Xenon is particularly attractive as a dark matter detector target for several reasons. It has a nuclear mass that is well matched to the preferred neutralino mass range. It scintillates in both the liquid and gas phases. It has a useful electron drift lifetime in both liquid and gas phases and can be used in a proportional ionisation mode. Two separable isotopes exist, one with spin and one without. However, it does have some disadvantages, such as: one needs a high level of purity, liquid xenon is more difficult to handle than a crystal scintillator, its scintillation signals are well in the UV $(\sim 1750 \AA)$, and its scintillation signals are very fast (<50 ns).

Figure 11] shows one proposed type of configuration for a two-phase system in which photomultipliers are used to record two scintillation signals for each event, S1 and S2 [35]. S1 is the primary scintillation signal from the liquid volume, which occurs as a direct result of the WIMP $/ \gamma$-ray scattering interaction. In addition to scintillation, the interaction will also produce localised ionisation in the liquid. An applied electric field is then used to drift the ionisation electrons towards and into the gaseous xenon. In the gas there is a region in which the applied electric field is strong enough to produce secondary scintillation, or electroluminescence, which produces signal S2. S1 and S2 are thus separated in time. At low electric field the S1 signal itself will be amenable to pulse shape analysis as described above for NaI. The S2 signal amplitude will depend on how many ionisation charges are drifted into the gas volume. This will depend on how many are produced in the initial interaction and on what fraction of those immediately recombine. The level of recombination is expected to be higher for events with a higher linear energy density deposit $d E / d x$, and so nuclear recoil type events are expected to show a much lower fraction of surviving drifting electrons. Hence, the ratio of S2 to S1 should be much lower for nuclear

Living Reviews in Relativity (2002-4)

http://www.livingreviews.org 


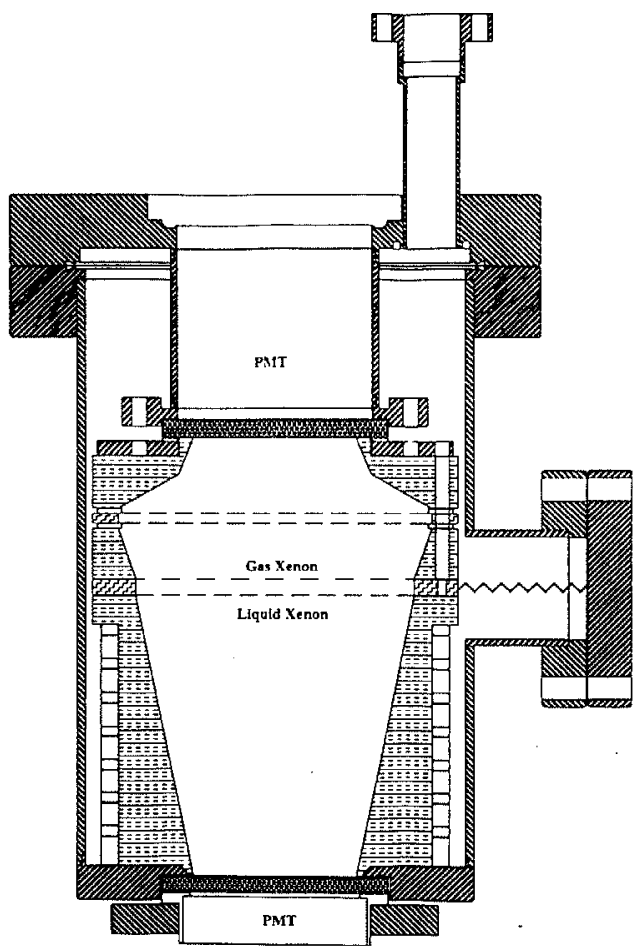

Figure 11: The two-phase xenon test chamber used by Wang [35]. 
recoils compared to say $\gamma$-ray deposits of the same amount. This effect has been demonstrated in low field operation [35, 69, and the left-hand panel of figure 12 shows some results from the chamber of figure 11. A $30 \mathrm{~kg}$ detector is being constructed [35] in which nuclear recoil events are identified by the lack of a secondary signal. An alternative scheme uses high-field operation in which ionisation from nuclear recoils can also be seen, and in which discrimination relies on the finite ratio of S2 to S1 [7]. This should give much higher background rejection and a $8 \mathrm{~kg}$ instrument is underway [135].
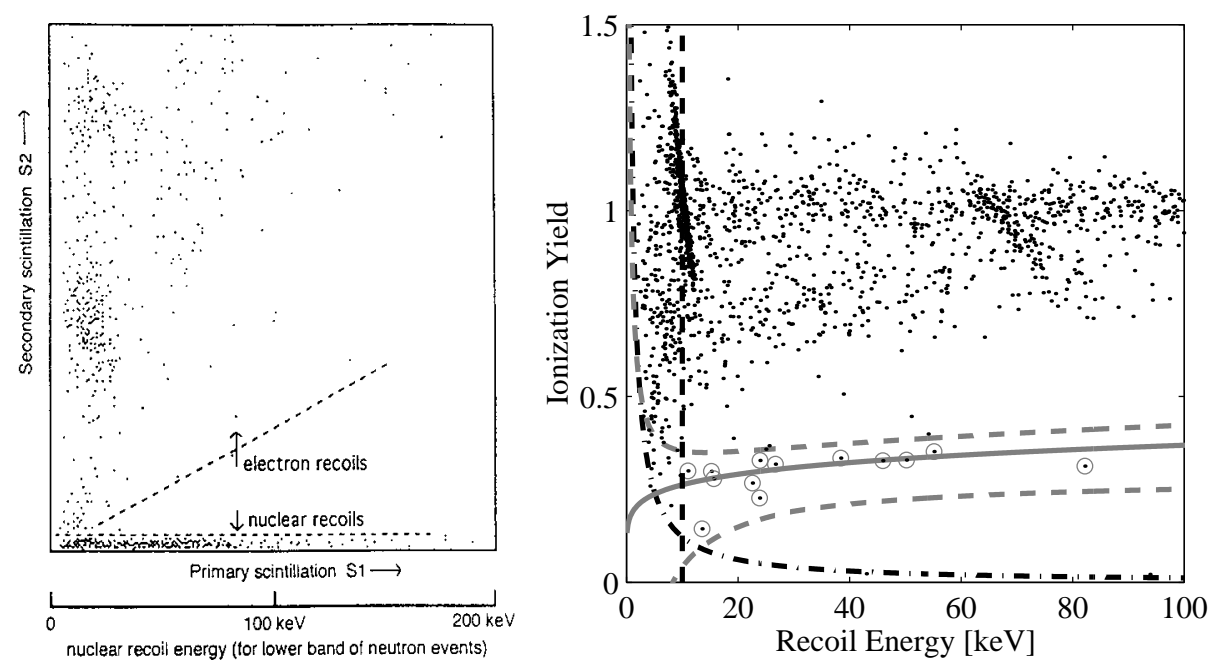

Figure 12: Relative signal amplitudes for $\gamma$-rays and neutrons for two hybrid type experiments. On the left is shown the primary to secondary scintillation signal amplitudes for a two-phase xenon instrument [35]. Neutrons have large S1/S2 ratios. The right hand panel shows the ionisation versus phonon performance of the CDMS germanium bolometer [3]. $\gamma$-rays populate the plot above the solid line with nuclear recoils below it. The circled points are experimental data thought to originate from neutron recoils.

The potential discrimination power available using the various techniques can be described by a figure of merit [117] as shown in figure 13. The top curves show the situation using pulse shape discrimination in NaI, and the two lower curves then show what improvement might be expected from using pulse height ratios from cooled NaI (UVIS) and a two-phase xenon system. In this figure, the performance improves as the figure of merit decreases and the potential advantage of liquid xenon over $\mathrm{NaI}$ is significant.

A variant on the above scheme is to try to 'image' the ionisation charge distribution using TEA (or TMA) added to the liquid xenon, which will convert scintillation photons into electrons [142, 101]. The idea here is that for nuclear recoil events there will be relatively few direct ionisation electrons left, due to the high $d E / d x$, and most drifting electrons will have been produced by

Living Reviews in Relativity (2002-4)

http://www.livingreviews.org 
photon absorption in the TEA/TMA. This should give an exponential spatial distribution (scale length around $2 \mathrm{~cm}$ ) of electrons drifting into the gas region. Whereas, for background $\gamma$-rays, there will be a significant core of electrons left over from the primary interaction in addition to those created by photon absorption, giving a more centrally peaked image.

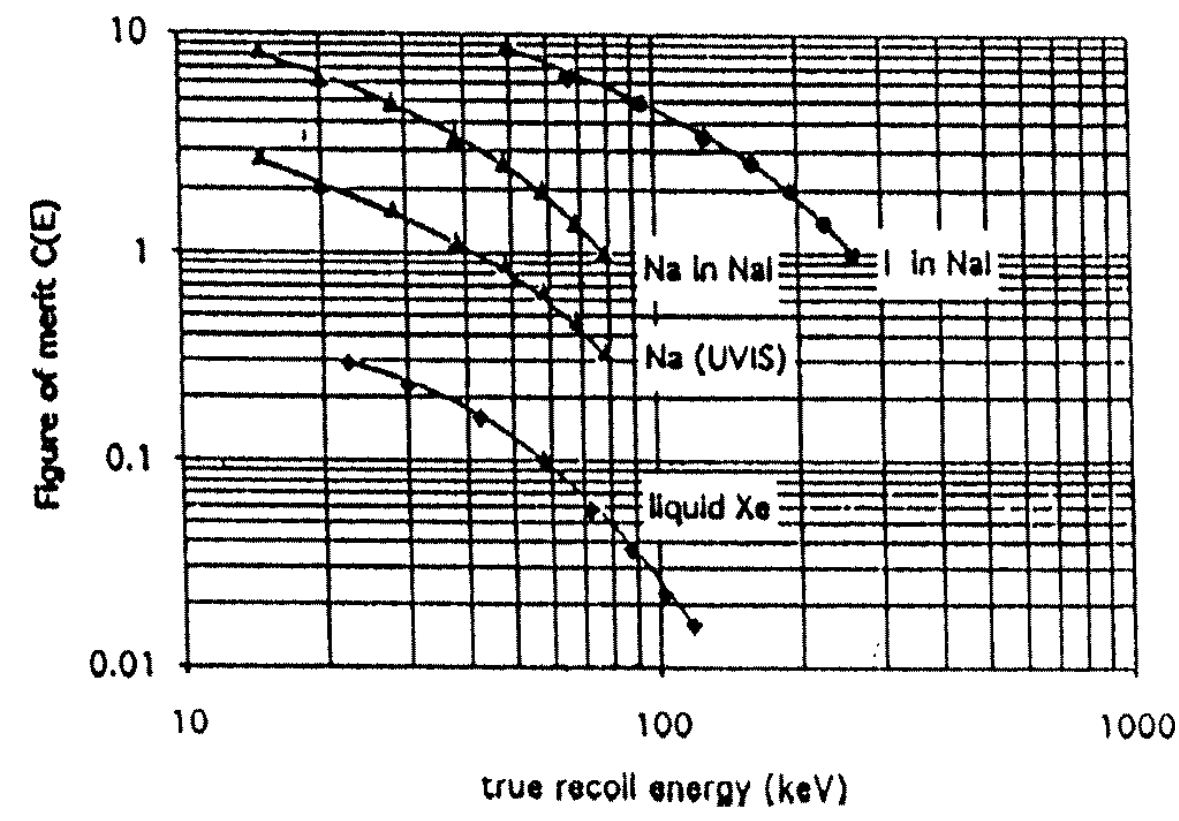

Figure 13: Relative figures of merit for the discrimination potential in NaI, cooled NaI and two-phase liquid xenon [117]. In this plot a lower figure of merit implies proportionately better performance.

\subsubsection{Phonon Detectors - Bolometers}

Most of the energy imparted to a recoiling nucleus during a WIMP scattering will ultimately end up as phonons. These can be detected as a temperature rise. For crystalline target materials the specific heat at low temperatures varies as $T^{3}$, so the lower the temperature the greater the temperature rise as $\Delta T \propto 1 / T^{3}$. In principle this should yield very good energy resolution limited by the statistical fluctuations in the numbers of phonons produced. At a temperature of $20 \mathrm{mK}$, a $1 \mathrm{~kg}$ detector could achieve $100 \mathrm{eV}$ resolution, with a correspondingly low threshold. However, in practise, the resolution is limited partly by the efficiency of the phonon 'cooling' process, whereby the initial non-thermal phonons with energies of $10^{-3}$ to $10^{-2} \mathrm{eV}$ become degraded into thermal phonons of around $10^{-5} \mathrm{eV}$. Once thermalised, the phonons then need to be coupled into the temperature sensors, which tend to be separate components bonded onto the target materials. For a $1 \mathrm{~kg}$ detector the temperature 
rise would be $\sim 10^{-7} \mathrm{~K} / \mathrm{keV}$, dependent on the Debye temperature of the material, and temperature sensors with this level of sensitivity at such very low temperatures are difficult to achieve. The earliest sensors used were doped semiconductors, such as NTD germanium. There are now more sensitive sensors available. These rely on superconducting transitions and two types are in use. One is the superconducting phase transition (SPT) thermometer [39, the other is the superconducting transition edge sensor (TES) [34]. In both cases the temperature rise is measured by monitoring movement along the transition from superconductor to normal metal. For the TES this provides a sensitivity to the higher energy phonons. As these have not suffered the extensive scattering needed to thermalise them, some positional information can be recovered.

\subsubsection{Hybrid Detectors Using Phonons and Scintillation/Ionisation}

The use of a separate temperature sensor bonded onto a target allows a range of different material choices for the target.

If semiconductor target materials are used, it is possible also to extract ionisation signals from bolometer experiments [121, 33. Nuclear recoils produce less ionisation compared to thermal energy than x-ray and $\gamma$-ray background events. For events initiated well away from surfaces, this allows for good discrimination power. Surface events, from external electrons for example, can be problematic as the ionisation can be inefficiently collected compared to the thermal energy, which mimics nuclear recoil signals. The ionisation signals are collected using charge-sensitive preamplifiers in the usual way for semiconductor diodes.

If scintillator target materials are used it is possible also to extract scintillation signals [39]. The situation is analogous to the simultaneous ionisation measurement in that nuclear recoil events are much less efficient at producing ionisation and excitation than typical background events. In this case it is even possible to use SPTs deposited on light absorbers (e.g. silicon) as the scintillation signal channel [39].

\subsubsection{Other Techniques}

Three other techniques are worthy of mention here. Two are techniques currently being developed while the third has been in use for some time. The first is the use of a gas target within a time projection chamber. The aim here is to image tracks of interactions within the time projection chamber and measure the range of the ionisation track and the energy deposition 24]. Nuclear recoil events have already been successfully recorded in a prototype device, and these have much shorter track lengths than an electron recoil of the same energy. This technique offers the prospect of realising a fully direction-sensitive detector that would not only enable use of all the directional WIMP signatures in attempting to extract signals, but would also allow the local WIMP velocity distribution to be measured. The second technique in this section is the use of superheated droplet detectors in which events leaving a high $d E / d x$ deposit are capable of vapourising the droplets 37,65 . Such detectors operate close to room temper-

Living Reviews in Relativity (2002-4)

http://www.livingreviews .org 
ature, exhibit low thresholds, and are insensitive to $\gamma$-rays that do not leave a sufficiently high density track. Readout can be either optical or acoustic. Finally, the ancient mica technique has already been used to derive upper limits to interaction cross-sections [10. Ancient mica contains an historical record of nuclear recoil interactions over exposure times of Gyrs. The defects left in the crystal can be etched and examined using an atomic force microscope. Defects left from natural radioactive processes will tend to leave much more pronounced etch pits than expected for a WIMP, so the technique involves looking at the size distribution of the etch pits.

Living Reviews in Relativity (2002-4) http://www.livingreviews.org 


\section{Neutralino Project Overview and Limits}

The range and breadth of experimental work now underway world-wide makes it very difficult to maintain an up-to-date and complete catalogue of activities. Table 3 presents an overview of experiments currently going on, but is far from exhaustive.

Figure 14, adapted from [23], shows the published upper limits on the nucleon-WIMP scattering cross-section for coherent and axial coupling respectively, as they were in 1996. In both cases, the best limits came from the large mass DAMA NaI experiments with the UKDMC NaI experiment close behind. For the coherent interactions the germanium results are comparable to the UKDMC NaI. Finally, it is intriguing that both the DAMA and UK NaI experiments have low-level signal effects that do not appear to be consistent with $\gamma$-ray backgrounds. The UKDMC experiment, using pulse-shape analysis, revealed a family of short-time-constant events [120, which are even faster than their neutron induced recoil events. The derived 'recoil spectrum' for these events falls with energy as expected for WIMPs (but the implied WIMP mass is rather high), and there is even some hint of an annual modulation. However, the effect has now been shown to be a spurious surface effect [125]. The DAMA experiment has an annual modulation signal, which has persisted in a consistent way through a complete rebuild of the experiment [20] and with data accumulated over a four year period [16. From their data it is possible to delineate an allowed region in coherent cross-section parameter space that contains cosmologically interesting combinations of MSSM parameters, as shown in figure 15 .

Finally, figure 16 shows the two most recent limits plots. The left hand plot comes from the CDMS collaboration [3], which runs a hybrid bolometer/ionisation experiment. The limit they have produced almost excludes completely the DAMA coherent cross-section result (shown as the solid grey region). However, CDMS operates in a shallow site and experiences an ambient neutron background. Their limit relies on the assumption that the circled events shown in figure 12 are indeed due to ambient neutrons. The numbers are consistent with this and there is a cross-check using multi-site multiple neutron scattering events. However, with such small numbers the result cannot be decisive. Recently, the EDELWEISS experiment has produced an upper limit that is comparable to CDMS for high WIMP masses [14]. The technique is similar to CDMS, but has the advantage of no detectable neutron background as it is in a deeper site. Unfortunately, it has a higher energy threshold at present and work is ongoing to improve this. So we are left with a tantalising claim of a WIMP annual modulation signal from DAMA, which they have defended despite much concern over possibilities of spurious annual modulation effects, and an almost exclusive limit from CDMS, which is statistically arguable. The right-hand plot in figure 16 shows a plot from the SIMPLE [38] experiment using superheated droplets. The target has non-zero spin; they present results in terms of limits to a pure spin-dependent cross-section. The plot shows a compilation of limits from all the other experiments that have reported to date. In the bottom left-hand

Living Reviews in Relativity (2002-4)

http://www. livingreviews .org 


\begin{tabular}{|c|c|c|c|c|c|}
\hline Experiment & Type & Target & $\begin{array}{l}\text { Quenching } \\
\text { factor }\end{array}$ & $\begin{array}{l}\text { Mass } \\
{[\mathrm{kg}]}\end{array}$ & $\mathrm{kg} \cdot$ days \\
\hline Heidelberg/Moscow & I & $\mathrm{Ge}$ & \multirow{3}{*}{0.25} & 2.88 & $>165$ \\
\hline HDMS & I & Ge & & 0.20 & - \\
\hline GENIUS & I & Ge & & 1000 & - \\
\hline TANDAR/USC/ & $\mathrm{I}$ & $\mathrm{Ge}$ & \multirow[t]{2}{*}{-} & 1.033 & \multirow[t]{2}{*}{831} \\
\hline PNL/Zaragoza & $\mathrm{S}$ & $\mathrm{NaI}$ & & 32 & \\
\hline $\begin{array}{c}\text { USC PNL Zaragoza } \\
\text { COSME/TWIN }\end{array}$ & $\mathrm{I}$ & $\mathrm{Ge}$ & & & \\
\hline $\begin{array}{c}\text { Neuchatel Caltech } \\
\text { PSI }\end{array}$ & $\mathrm{I}$ & $\mathrm{Ge}$ & & & \\
\hline \multirow{3}{*}{ UKDMC } & $\mathrm{S}$ & $\mathrm{NaI}(\mathrm{Tl})$ & $\mathrm{Na}(0.3)$ & 6 & $>1500$ \\
\hline & S & $\mathrm{Xe}$ & $\mathrm{I}(0.08)$ & 6 & - \\
\hline & $\mathrm{S} / \mathrm{I}$ & $\mathrm{Xe}$ & $\mathrm{Xe}(0.2)$ & 20 & - \\
\hline \multirow{3}{*}{ DAMA } & $\mathrm{S}$ & $\mathrm{NaI}(\mathrm{Tl})$ & $\mathrm{Ca}(0.08)$ & 115 & 30000 \\
\hline & S & $\mathrm{CaF}_{2}$ & $\mathrm{~F}(0.12)$ & 0.37 & 10 \\
\hline & S & $\mathrm{Xe}$ & $\mathrm{Xe}(0.65)$ & 6.5 & 823 \\
\hline ELEGANT-V & $\mathrm{S}$ & $\mathrm{NaI}(\mathrm{Tl})$ & & 662 & 241630 \\
\hline ELEGANT-VI & $\mathrm{S}$ & $\mathrm{CaF}_{2}$ & & 8 & \\
\hline Saclay & $\mathrm{S}$ & $\mathrm{NaI}$ & & & \\
\hline Amherst UCB & $\mathrm{O}$ & Mica & - & - & 1 Gyr \\
\hline $\begin{array}{c}\text { SIMPLE } \\
\text { (CERN Lisbon Paris) }\end{array}$ & $\mathrm{O}$ & Freon & & 1 & 0.190 \\
\hline Montreal Chalk River & $\mathrm{O}$ & $\mathrm{F}, \mathrm{Cl}$ & & 1 & \\
\hline $\begin{array}{c}\text { Tokyo Dark Matter } \\
\text { Search }\end{array}$ & $\mathrm{P}$ & $\mathrm{LiF}$ & 1 & 0.168 & \\
\hline Milano & $\mathrm{P}$ & $\mathrm{TeO}_{2}$ & & 7 & \\
\hline ROSEBUD & $\mathrm{P}$ & Sapphire & & 0.100 & \\
\hline CRREST & $\mathrm{P}$ & Sapphire & & 0.262 & \\
\hline CMDS & $\mathrm{P} / \mathrm{I}$ & $\mathrm{Ge}$ & & 0.262 & \\
\hline EDELWEISS & $\mathrm{P} / \mathrm{I}$ & $\mathrm{Ge}$ & & 0.900 & \\
\hline Orpheus & $\mathrm{O}$ & $\mathrm{Sn}$ & & 0.032 & \\
\hline Salopard & $\mathrm{O}$ & Sn & & 0.100 & \\
\hline
\end{tabular}

Table 3: Experiment Overview. 
corner of this plot can be seen the "tip of the iceberg" of MSSM predictions for spin-dependent cross-sections.

The need for more sensitive and more powerful experiments is clear as we start to impinge more and more on the allowed neutralino parameter space and as experiments begin to reveal features at levels never before investigated. The need for multiple experiments to confirm the results of others is also clear.

Living Reviews in Relativity (2002-4)

http://www.livingreviews.org 

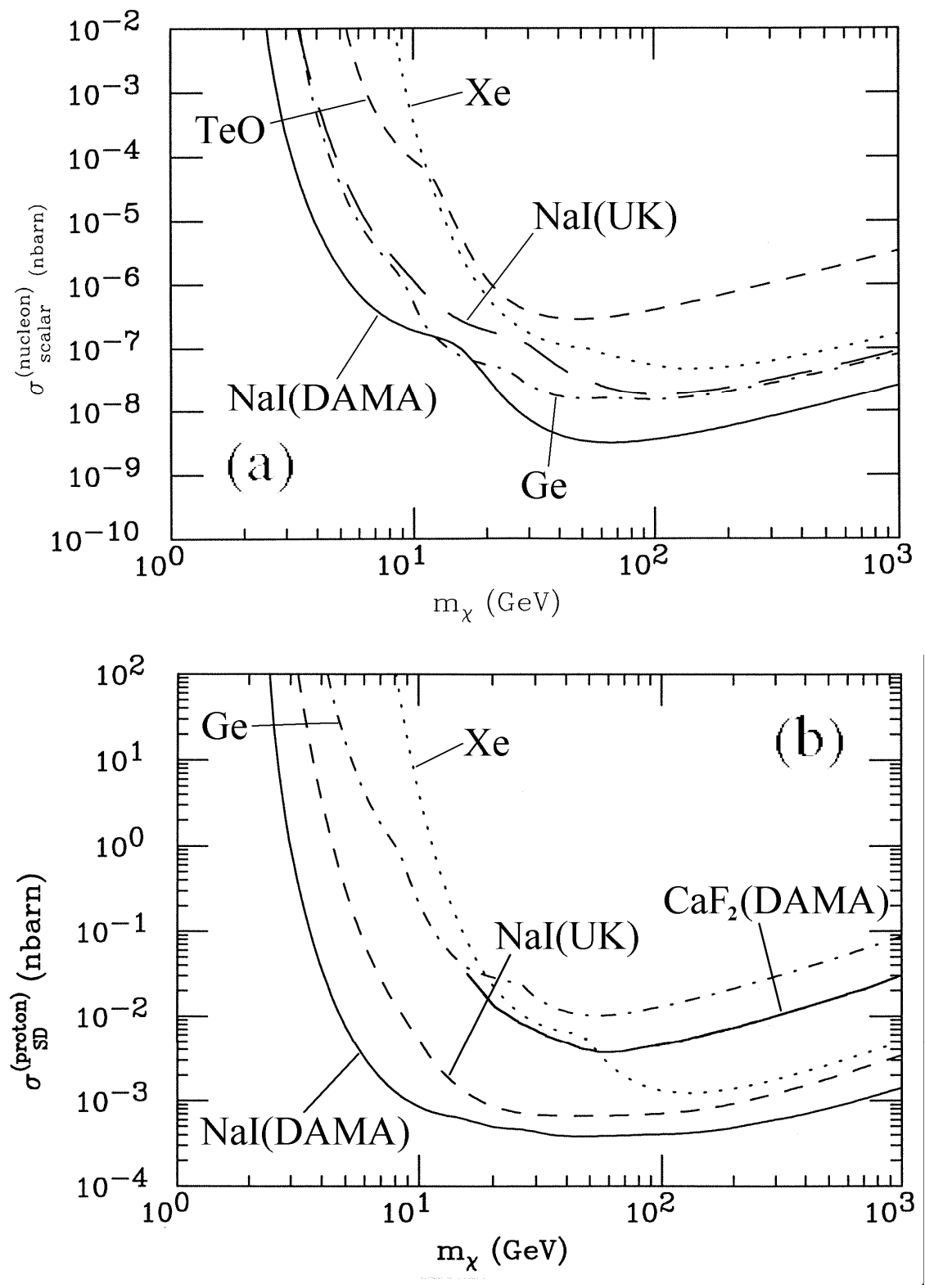

Figure 14: Latest published upper limits on ( $a$ - upper panel) coherent and ( $b-$ lower panel) axial coupled WIMP-nucleon cross-sections adapted from [23]. 

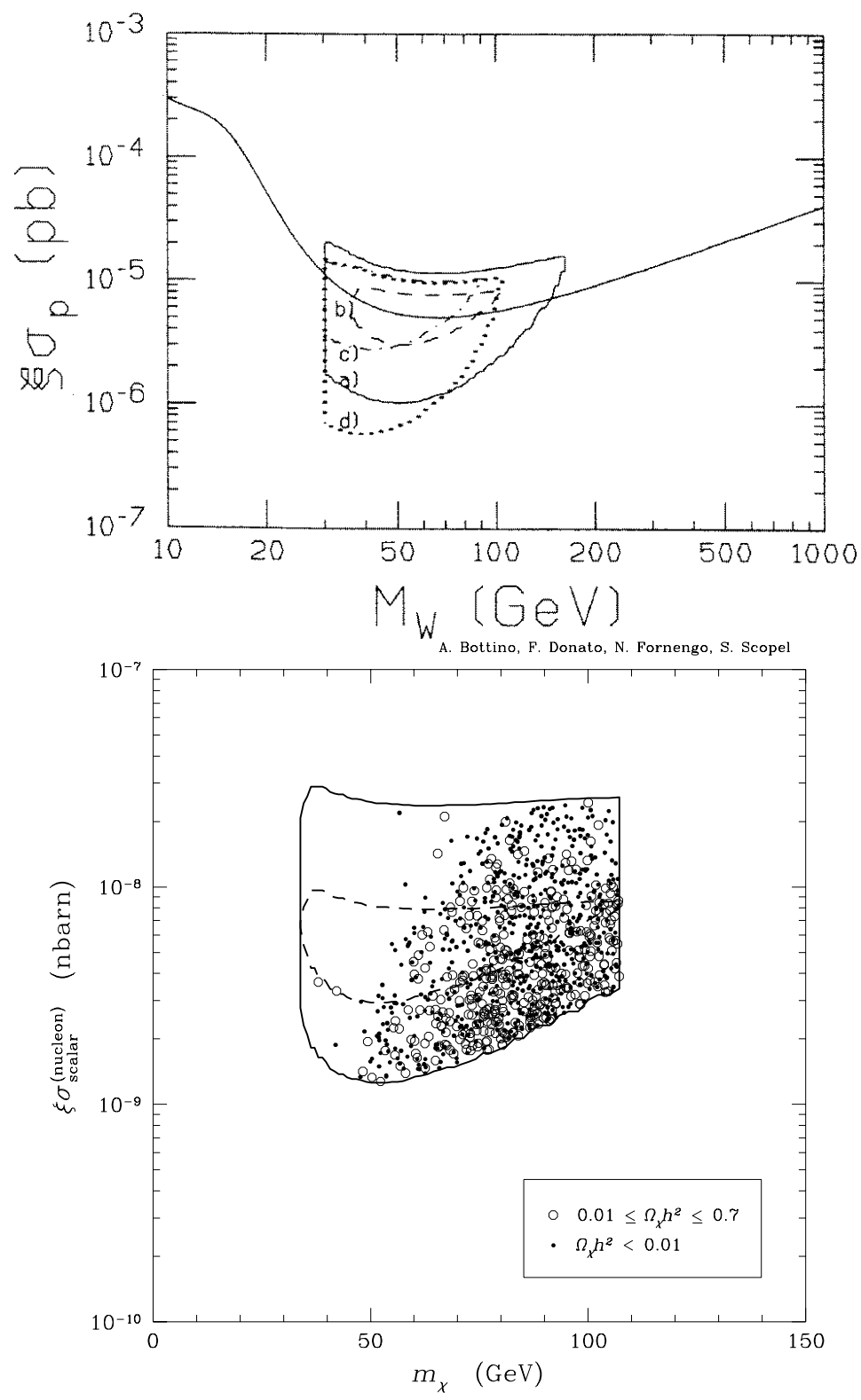

Figure 15: The upper panel shows the region of coherent cross-section parameter space consistent with the DAMA NaI annual modulation results [16]. The four curves show the results from each individual year of the four year period shown in figure 8. The lower panel shows a scatter plot of possible MSSM models which populate the region defined by the first two years of data from [22]. Open circles are cosmologically interesting.

Living Reviews in Relativity (2002-4) http://www.livingreviews.org 

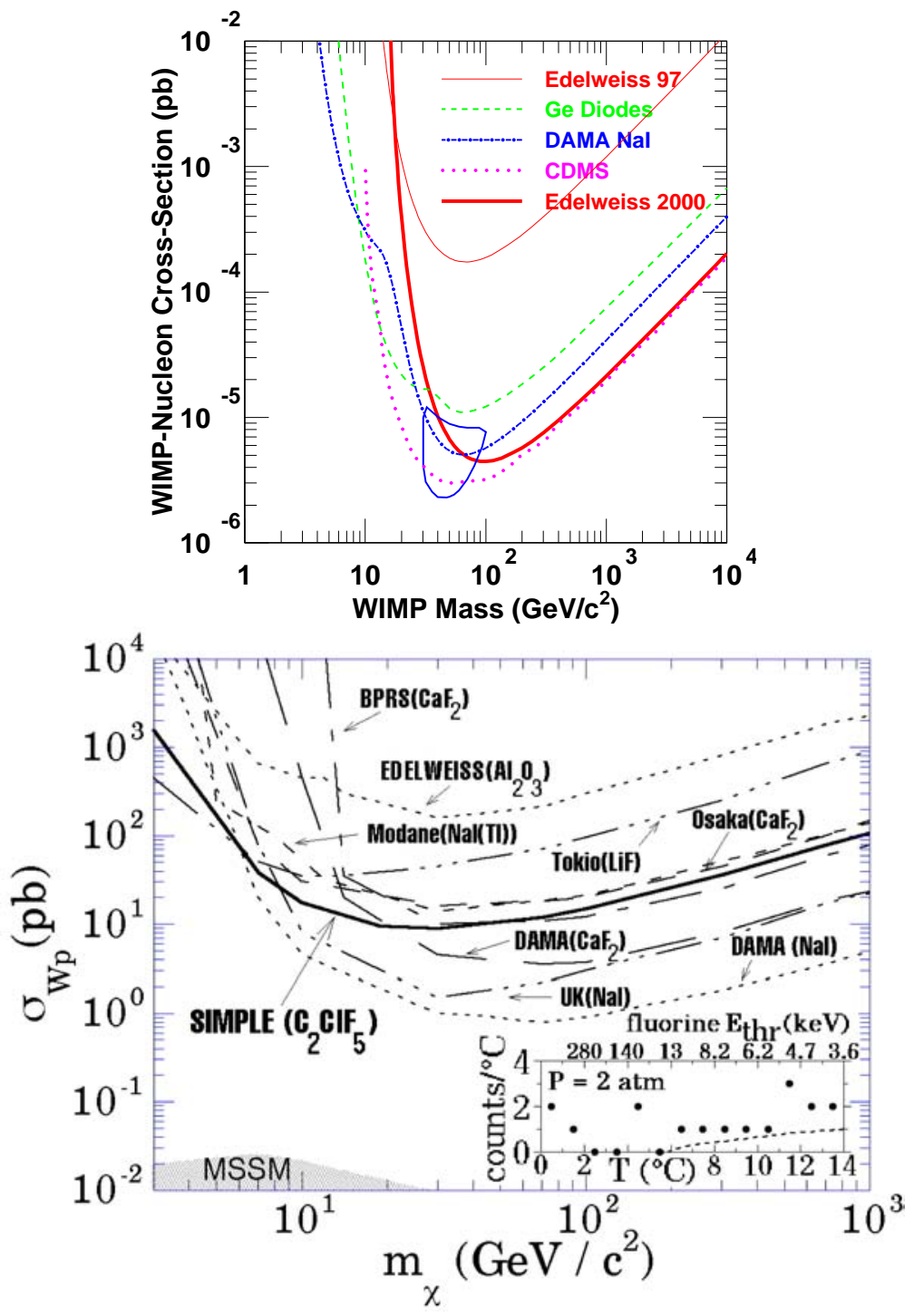

Figure 16: The upper panel shows the current results on the allowed coherent cross-section parameter space. The plot is from [14] and shows the CDMS $3 \sigma$ upper limit [3] (dotted purple curve), the DAMA annual modulation positive detection region (blue solid curve), the DAMA upper limit from pulse shape discrimination (blue dot-dash curve), the EDELWEISS limits [14] (red curves) and the current limits from all combined germanium ionisation detectors [70] (dashed green curve). The lower panel shows an equivalent plot for the axial spin-dependent coupling cross-section. This is a composite plot produced by the SIMPLE collaboration in announcing their latest result [38].

Living Reviews in Relativity (2002-4) http://www.livingreviews.org 


\section{Future Promises and Prospects}

Given the level of current activity it is inevitable that over the next several years there will be substantial advances in "the dark matter problem." The main issues will be:

- Refinement/Revision of the Standard Cosmological Model using:

- New observational data on CMB anisotropies, particularly the second peak. Definitive data should come from the ESA PLANCK mission due for launch in 2007. Balloon-borne instruments may also be useful.

- New observational data on large-scale structure from galaxy redshift surveys.

- Improved statistics on high-redshift Type Ia supernova. This is needed to provide proper confirmation of their properties and their use as standard candles and also to define the distribution more clearly.

- Further work on non-standard BBN models, such as those invoking degenerate neutrino species.

- Continued theoretical modelling of the CMB anisotropies using cosmological models. Although this appears to have been developed to a fine art, there must be avenues for new development.

- Investigation of alternative types of dark matter, such as "warm dark matter." Further experimental data on the neutrino masses would be relevant here as well, although the recent evidence for neutrino mixing from SNO suggests that the neutrino masses are unlikely to be cosmologically significant [5].

- Refinement of Galaxy Formation and Structure Models using:

- Continued high-resolution $N$-body simulations including more refined feedback on baryonic condensation, star formation, and massive black-hole formation.

- High-resolution rotation curve measurements. These will help to establish the central density distributions and will also look for modulations at larger radii to assess dark matter infall models.

- High-resolution $N$-body simulations to establish CDM dynamics and structure within galaxy halos.

- Continued observational searches for cold baryonic dark matter, using microlensing or infra-red, sub-millimeter observations of small clouds.

- Experimental Searches for Neutralinos, including:

- Improved versions of CDMS, CCREST, and UKDMC experiments in the next two years, which will extend significantly below the DAMA region and resolve whether neutralinos have already been observed or not.

Living Reviews in Relativity (2002-4)

http://www. livingreviews .org 
- Several other experiments, such as GENIUS [76, that may also come online in the next several years. In fact, within about 6 years it is just possible that the whole of the parameter space in figure 6 will have been explored.

- A number of indirect search experiments may produce useful complementary data, such as neutrino telescopes, $\gamma$-ray missions (GLAST 89]), or particle experiments (AMS).

- Detectors with directional response will have been developed at the prototype level, ready to become neutralino "telescopes" should the need arise.

- Supersymmetry and Supergravity will have independent input from:

- The Large Hadron Collider, which will begin operation in 5-6 years time. Within a few years of data taking it should start to constrain much of the MSSM and SUGRA parameter space.

- Continued exploration of MSSM and SUGRA models to refine calculations of scattering cross-sections.

Of course, the most satisfying scientific output would be the discovery of the neutralino as the dominant dark matter component of the Milky Way. The prospects for this are very good. Figure 17 shows two panels taken from 49] in which the likely search areas to be completed by 2006 are delineated. The parameter space chosen for these plots has the universal scalar mass $m_{0}$ and the gaugino mass $M_{1 / 2}$ as the coordinates. The two plots correspond to two illustrative values of $\tan \beta$. The solid dark green regions are already excluded. In the light green/yellow shaded area $0.025 \leq \Omega_{\mathrm{m}} \leq 1$, while in the blue shaded area $0.1 \leq \Omega_{\mathrm{m}} \leq 0.3$. The curves then show which regions of parameter space are likely to be addressed over the coming years. For each curve the forthcoming experiments will search the region between the curve and the dark green area. The red curve corresponds to the direct search techniques such as the next generation of CDMS, CCREST and UKDMC Xenon experiments. This curve should be reached in 2-3 years time. Following that, there are already larger, better experiments being planned that could push further still [133. The other curves shown correspond to various indirect search techniques, including $\gamma$-rays, neutrinos, and positrons. Feng et al. [49] describe the situation in great detail. The complementarity of the various techniques is apparent and multiple detections would provide a powerful diagnostic of SUSY parameters.

The scientific impact of a positive neutralino detection would extend not only to cosmology and astrophysics in almost every aspect, but would also be of the utmost importance to supersymmetry and fundamental physics. If, in addition to the neutralino, the axion is also implicated, then we will have a double bonanza, which also will verify the adopted solution to $\mathrm{CP}$ violation. Seldom has there been a problem that impinges on so many fundamentally important issues and this justifies the current level of activity on all fronts. The next several years promise to be very interesting.

Living Reviews in Relativity (2002-4) http://www.livingreviews.org 

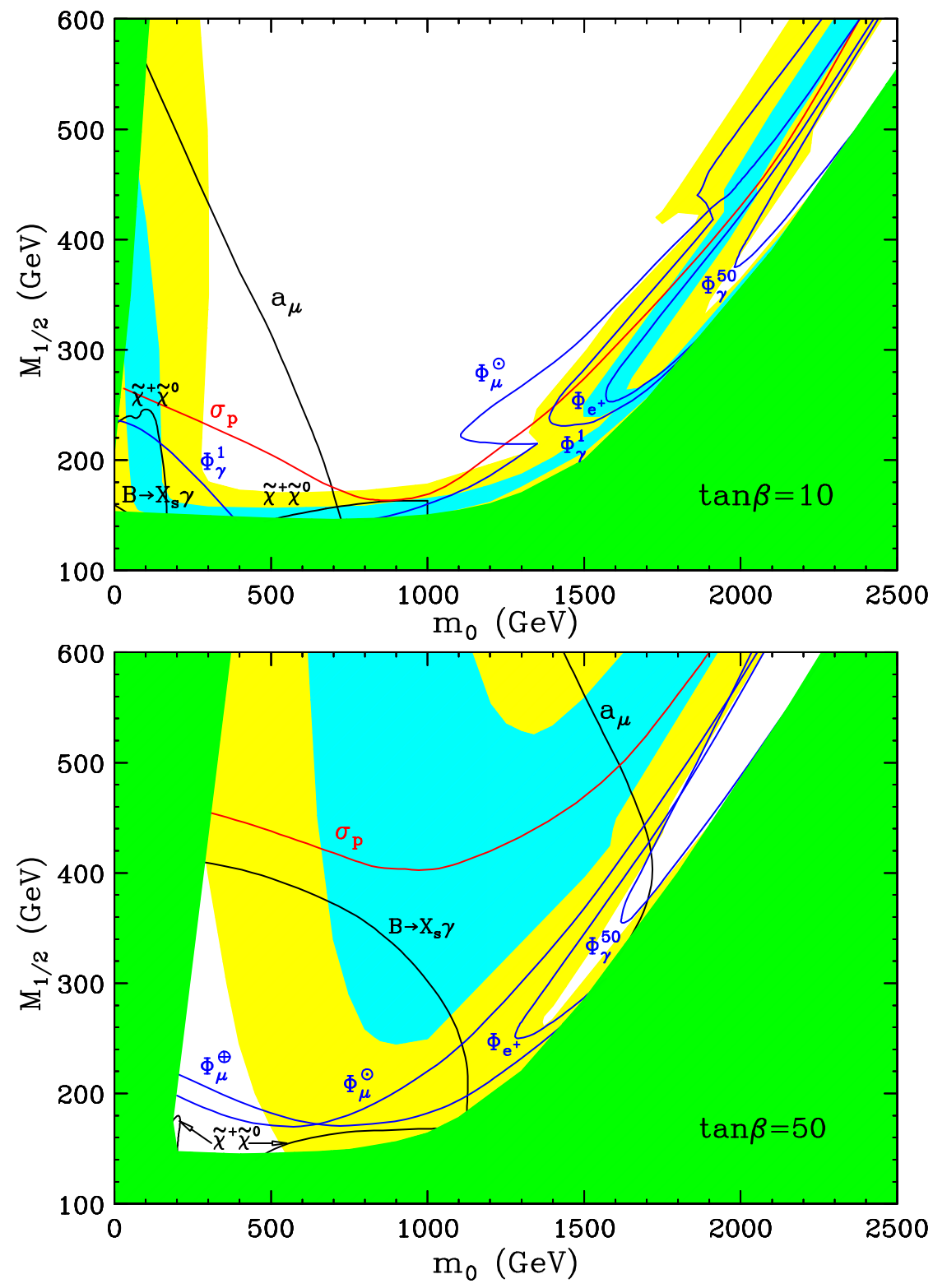

Figure 17: Expected progress in covering MSSM parameter space from both indirect and direct search techniques over the next several years [49].

Living Reviews in Relativity (2002-4) http://www. livingreviews .org 


\section{References}

[1] Abriola, D., Avignone, D.T., Brodzinski, R.L., Collar, J.I., Di Gregorio, D.E., Farach, H., Garcia, E., Gattone, A.O., Hasenbalg, F., Huck, H., Miley, H.S., Morales, A., Morales, J., Ortiz de Solorzano, A., Puimedon, J., Reeves, J.H., Saenz, C., Salinas, A., Sarsa, M.L., Tomasi, D., Urteaga, I., and Villar, J.A., "Search for Dark Matter Signals in the Southern Hemisphere", in Spooner, N.J.C., ed., Proc. of 1st Workshop on The Identification of Dark Matter, 403-408, (World Scientific, Singapore, 1997). 7. $5.4 .1,8$

[2] Abriola, D., Avignone, F.T., Brodzinskic, R.L., Collar, J.I., Di Gregorio, D.E., Farach, H.A., Garcia, E., Gattone, A.O., Guerard, C.K., Hasenbalg, F., Huck, H., Miley, H.S., Morales, A., Morales, J., Ortiz de Solorzano, A., Puidemon, J., Reeves, J.H., Salinas, A., Sarsa, M.I., and Villar, J.A., "Search for an Annual Modulation of Dark Matter Signals with a Germanium Spectrometer at the Sierra Grande Laboratory", Astropart. Phys., 10, 133-139, (1999). For a related online version see: D. Abriola, et al., "Search for an Annual Modulation of Dark Matter Signals with a Germanium Spectrometer at the Sierra Grande Laboratory", (September, 1998), [Online Los Alamos Archive Preprint]: cited on 7 July 2001, http://xxx.lanl.gov/abs/astro-ph/9809018 5.4.1

[3] Abusaidi, R., et al. (CDMS collaboration), "Exclusion Limits on the WIMP-Nucleon Cross-Section from the Cryogenic Dark Matter Search", Phys. Rev. Lett., 84, 5699-5703, (2000). For a related online version see: et al. (CDMS collaboration) Abusaidi, R., "Exclusion Limits on the WIMP-Nucleon Cross-Section from the Cryogenic Dark Matter Search", (March, 2000), [Online Los Alamos Archive Preprint]: cited on 7 August 2000, http://xxx.lanl.gov/abs/astro-ph/0002471, 12, 6, 16

[4] Ahlen, S., Balebanov, V.M., Battiston, R., Becker, U., Burger, J., Capell, M., Chen, H.F., Chen, H.S., Chen, M., Chernoplekov, N., Clare, R., Dai, T.S., De Rujula, A., Fisher, P., Galaktinov, N., Gougas, A., Wen-Qi, G., He, M., Koutsenko, V., Lebedev, A., Li, T.P., Lu, Y.S., Luckey, D., Ma, Y., McNeil, R., Orava, R., Prevsner, A., Plyskine, V., Rubinstein, H., Sagdeev, R., Salomon, M., Tang, H.W., Ting, S.C.C., Vetlitsky, I., Wang, Y.F., Ping-Chou, X., Xu, Z.Z., Wefel, J.P., Zhang, Z.P., Zhou, B., and Zichichi, A., "An antimatter spectrometer in space", Nucl. Instrum. Methods, A350(1), 351-367, (1994). 4.1.

[5] Ahmad, Q.R., et al. (SNO Collaboration), "Measurement of the rate of $\nu_{e}+d \rightarrow p+p+e^{-}$interactions produced by ${ }^{8} \mathrm{~B}$ solar neutrinos at the Sudbury Neutrino Observatory", Phys. Rev. Lett., 87, 071301-1-0713016 , (2001). For a related online version see: et al. (SNO Collaboration) Ahmad, Q.R., "Measurement of charged current interactions by ${ }^{8} \mathrm{~B}$ solar neutrinos at the Sudbury Neutrino Observatory", (June, 2001), [Online

Living Reviews in Relativity (2002-4) http: //www. livingreviews . org 
HTML document]: cited on 7 July 2001, http://www . sno.phy - queensu . ca/sno/first_results/page00.html. 7

[6] Akerib, D.S., "WIMP Searches with Low Temperature Detectors: Methods, Merits, and Challenges", in Spooner, N.J.C., and Krudryavtsev, V., eds., Proc. of 2nd Workshop on The Identification of Dark Matter, 241247, (World Scientific, Singapore, 1999). 4.2

[7] Akimov, D.Y., Bewick, A., Danilov, M.V., Davidge, D., Dawson, J., Howard, A.S., Jones, W.G., Kovalenko, A.G., Kovalenko, D.A., Lebedev, V.N., Quenby, J.J., and Sumner, T.J., "Development of a Two-Phase Xenon Discriminating Detector for use in Dark Matter Searches at the Boulby Underground Laboratory", in Cline, D.B., ed., Sources and Detection of Dark Matter and Dark Energy in the Universe - Fourth International Symposium, Proc. 4th International Symposium on Origins of Dark Matter, 461-468, (Springer-Verlag, Berlin and Heidelberg, 2001). 5.4 .3

[8] Alcock, C., Allsman, R.A., Alves, D.R., Axelrod, T.S., Becker, A.C., Bennett, D.P., Cook, K.H., Dalal, N., Drake, A.J., Freeman, K.C., Geha, M., Griest, K., Lehner, M.J., Marshall, S.L., Minniti, D., Nelson, C.A., Peterson, B.A., Popowski, P., Pratt, M.R., Quinn, P.J., Stubbs, C.W., Sutherland, W., Tomaney, A.B., Vandehei, T., and Collaboration), Welch D. (MACHO, "The MACHO Project: Microlensing Results from 5.7 Years of LMC Observations", Astrophys. J., 542, 281307, (2000). For a related online version see: C. Alcock, et al., "The MACHO Project: Microlensing Results from 5.7 Years of LMC Observations", (2000), [Online Los Alamos Archive Preprint]: cited on 14 August 2000, http://xxx.lanl.gov/abs/astro-ph/0001272. 3.1

[9] Balbi, A., Baccigalupi, C., Matarrese, S., Perrotta, F., and Vittorio, N., "Implications on Quintessence Models from MAXIMA-1 and BOOMERanG-98", Astrophys. J., 547, L89-L92, (2001). For a related online version see: A. Balbi, et al., "Implications on Quintessence Models from MAXIMA-1 and BOOMERanG-98", (September, 2000), [Online Los Alamos Archive Preprint]: cited on 27 September 2000, http: //xxx.lanl.gov/abs/astro-ph/0009432. 2.2

[10] Baltz, E.A., Westphal, A.J., and Snowden-Ifft, D.P., "Probing the Structure of the Cold Dark Matter Halo with Ancient Mica", Phys. Rev. D, 59, 023510-1-023510-5, (1999). 5.4.6

[11] Barton, J.C., Blair, I., and Edgington, J., "Pulse-Shape Analysis of Scintillation Signals from NaI", Submitted to Appl. Radiation 83 Isotopes, (1999). 5.4 .2

[12] Baudis, L., Hellmig, J., Klapdor-Kleingrothaus, H.V., Ramachers, Y., and Strecker, H., "Dark Matter Search with the HDMS-Experiment and the GENIUS Project", Nucl. Phys. B, 70, 106-110, (1999). 5.4.1

Living Reviews in Relativity (2002-4)

http://www.livingreviews.org 
[13] Beck, M., Bensch, F., Bockholt, J., Heusser, G., Klapdor-Kleingrothaus, H.V., Maier, B., Petry, F., Piepke, A., Strecker, H., Vollinger, M., Zuber, K., Balysh, A., Belyaev, S.T., Demehin, A., Gurov, A., Kondratenko, I., Lebedev, V.I., and Muller, A., "Searching for Dark Matter with the Enriched Ge Detectors of the Heidelberg-Moscow $\beta \beta$ Decay Experiment", Phys. Lett. B, 336(2), 141-146, (1994). 5.4.1

[14] Benoit, A., Berge, L., Broniatowski, A., Chambon, B., Chapellier, M., Chardin, G., Charvin, P., De Jesus, M., Di Stefano, P., Drain, D., Dumoulin, L., Gascon, J., Gerbier, G., Goldbach, C., Goyot, M., Gros, M., Hadjout, J.P., Juillard, A., de Lesquen, A., Loidl, M., Mallet, J., Marnieros, S., Martineau, O., Mirabolfathi, N., Mosca, L., Miramonti, L., Navick, X.-F., Nollez, G., Pari, P., Stern, M., and Vagneron, L. (EDELWEISS Collaboration), "First Results of the EDELWEISS WIMP Search using a 320 g Heat-and-Ionisation Ge Detector", Phys. Lett. B, 513, 15-22, (2001). For a related online version see: A. Benoit, et al., "First Results of the EDELWEISS WIMP Search using a 320g Heat-and-Ionisation Ge Detector", (June, 2001), [Online Los Alamos Archive Preprint]: cited on 7 July 2001, http://xxx.lanl.gov/abs/astro-ph/0106094. 6, 16

[15] Bernabei, R., Belli, P., Cerulli, R., Dai, C.J., Ignesti, G., Incicchitti, A., Montecchia, F., and Prosperi, D., "Improved Limits on WIMP- ${ }^{129}$ Xe Inelastic Scattering", New J. Phys., 2, 15.1-15.7, (2000). 5.2

[16] Bernabei, R., Belli, P., Cerulli, R., Montecchia, F., Amato, M., Ignesti, G., Incicchitti, A., Prosperi, D., Dai, C.J., He, H.L., Kuang, H.H., and Ma, J.M., "Search for WIMP Annual Modulation Signature: Results from DAMA/NaI-3 and DAMA/NaI-4 and the Global Combined Analysis", Phys. Lett. B, 480(1), 23-31, (2000). 5.4.1, 8, 5.4.2, 6, 15

[17] Bernabei, R., Belli, P., F., Montecchia, Di Nicolantonio, W., Incicchitti, A., Prosperi, D., Bacci, C., Dai, C.J., Ding, L.K., Kuang, H.H., and Ma, J.M., "WIMPs Search by Scintillators: Possible Strategy for Annual Modulation Search with Large-Mass Highly-Radiopure NaI(Tl)", Nucl. Phys. Proc. Suppl., 70, 79-84, (1999). For a related online version see: R. Bernabei, et al., "WIMPs Search by Scintillators: Possible Strategy for Annual Modulation Search with Large-Mass Highly-Radiopure NaI(Tl)", (1997), [Online Los Alamos Archive Preprint]: cited on $14 \mathrm{Au}-$ gust 2000, http://xxx.lanl.gov/abs/astro-ph/9710290. Report No.: ROM2F/97/33, ROM2F/97/39. 5.4 .2

[18] Bernabei, R., Belli, P., Landoni, V., Montecchia, F., Di Nicolatonio, W., Incicchitti, A., Prosperi, D., Bacci, C., and Dai, D.J., "Limits on WIMP129Xe inelastic scattering", Phys. Lett. B, 387(1), 222-226, (1996). 5.2

[19] Bernabei, R., Belli, P., Monecchia, F., Incicchitti, A., Di Nicolantonio, W., Prosperi, D., Bacci, C., Dai, C.J., Ding, L.K., Kuang, H.H., Ma, J.M., and Yao, Z.G., "Improved Limits on WIMP_ ${ }^{19}$ F Elastic Scattering

Living Reviews in Relativity (2002-4) http://www.livingreviews.org 
and First Limit on the $2 \mathrm{EC} 2 \nu^{40} \mathrm{Ca}$ Decay by Using a Low Radioactive $\mathrm{CaF}_{2}(\mathrm{Eu})$ Scintillator", Astropart. Phys., 7, 73-76, (1997). 5.4 .2

[20] Bernabei, R., Belli, P., Montecchia, F., Di Nicolantonio, W., Ignesti, G., Incicchitti, A., Prosperi, D., Dai, C.J., Ding, L.K., Kuang, H.H., and Ma, J.M., "On a Further Search for a Yearly Modulation of the Rate in Particle Dark Matter Direct Search", Phys. Lett. B, 450, 448-455, (1999). Report No.: ROM2F/98/34. 5.4.2, 6

[21] Boriello, A., and Salucci, P., "Cold Dark Matter Halos Must Burn", (June, 2001), [Online Los Alamos Archive Preprint]: cited on 7 July 2001, http://xxx.lanl.gov/abs/astro-ph/0106251. Former title: "Dark Halos around Spirals; no role for collisionless Cold Dark Matter particles". 2.3

[22] Bottino, A., Donato, F., Fornengo, N., and Scopel, S., "Combining Data of Annual Modulation Effect in WIMP Direct Detection with Measurements of WIMP Indirect Searches", Astropart. Phys., 10, 203-210, (1999). For a related online version see: A. Bottino, et al., "Combining Data of Annual Modulation Effect in WIMP Direct Detection with Measurements of WIMP Indirect Searches", (1998), [Online Los Alamos Archive Preprint]: cited on 14 August 2000, http://xxx.lanl.gov/abs/hep-ph/9809239. 15

[23] Bottino, A., Donato, F., Mignola, G., and Scopel, S., "Exploring the Supersymmetric Parameter Space by Direct Search for WIMPs", Phys. Lett. B, 402, 113-114, (1997). For a related online version see: A. Bottino, et al., "Exploring the Supersymmetric Parameter Space by Direct Search for WIMPs", (1996), [Online Los Alamos Archive Preprint]: cited on 14 August 2000, http://xxx.lanl.gov/abs/hep-ph/9612451. 6, 14

[24] Buckland, K.N., Lehner, M.J., and Masek, G.E., "Low-Pressure TPC for Dark Matter Searches: Future Directions", in Spooner, N.J.C., ed., Proc. of 1st Workshop on The Identification of Dark Matter, 475-480, (World Scientific, Singapore, 1997). 5.4.6

[25] Burkert, A., "Self-Interacting Cold Dark Matter Halos", (2000), [Online Los Alamos Archive Preprint]: cited on 7 July 2001, http://xxx.lanl. gov/abs/astro-ph/0012178. 2.3

[26] Burkert, A., and Silk, J., "On the structure and nature of dark matter halos", (1999), [Online Los Alamos Archive Preprint]: cited on 14 August 2000, http://xxx.lanl.gov/abs/astro-ph/9904159. 2.3

[27] Burles, S., Nollett, K.M., Truran, J.N., and Turner, M.S., "Sharpening the predictions of big-bang nucleosynthesis", Phys. Rev. Lett., 82, 4176-4179, (1999). For a related online version see: S. Burles, et al., "Sharpening the predictions of big-bang nucleosynthesis", (1999), [Online Los Alamos

Living Reviews in Relativity (2002-4)

http://www.livingreviews.org 
Archive Preprint]: cited on 14 August 2000, http://xxx.lanl.gov/abs/ astro-ph/9901157. 2.2, 2.2.

[28] Caldwell, D.O., "Direct Search for Relic Particles", in Bottino, A., Di Credico, A., and Monacelli, P., eds., TAUP97, Nucl. Phys. B (Proc. Suppl.) 70, 43-53, (Elsevier, Amsterdam, 1999). 5.4.1, 7

[29] Caldwell, R.R., Dave, R., and Steinhardt, P.J., "Cosmological Imprint of an Energy Component with General Equation of State", Phys. Rev. Lett., 80(8), 1582-1585, (1998). 2.2

[30] Carr, B.J., "Baryonic Dark Matter", Comments Astrophys., 14(5), 257280, (1990). 3, 2,3

[31] Carr, B.J., "Baryonic Dark Matter in Galaxies", in Menzies, J.W., and Sackett, P.D., eds., Microlensing 2000: A New Era of Microlensing Astrophysics, volume 239 of ASP Conf. Proc. Ser., 37-52, (ASP, San Francisco, 2001). For a related online version see: B.J. Carr, "Baryonic Dark Matter in Galaxies", (2000), [Online Los Alamos Archive Preprint]: cited on 7 July 2001, http://xxx.lanl.gov/abs/astro-ph/0008028. 3, 3.1

[32] Carr, B.J., and Sakellariadou, M., "Dynamical Contraints on Dark Matter in Compact Objects", Astrophys. J., 516(1), 195-220, (1998). 3.1

[33] Chardin, G., "Preliminary Results of the EDELWEISS Experiment", in Spooner, N.J.C., and Krudryavtsev, V., eds., Proc. of 2nd Workshop on The Identification of Dark Matter, 365-370, (World Scientific, Singapore, 1999). 5.4 .5

[34] Clarke, R.M. (For the CDMS Collaboration), "Results of the Cryogenic Dark Matter Search (CDMS) Obtained Using a New Athermal Phonon Mediated Detector", in Spooner, N.J.C., and Krudryavtsev, V., eds., Proc. of 2nd Workshop on The Identification of Dark Matter, 353-358, (World Scientific, Singapore, 1999). 5.4.4

[35] Cline, D., Curioni, A., Lamarina, A., Mannochi, G., Otwinowski, S., Periale, L., Periale, R., Picchi, P., Pietropaolo, F., Wang, H., and Woo, J., "A WIMP Detector with Two-Phase Xenon", Astropart. Phys., 12(4), 373-377, (2000). $5.4 .3,11,12$

[36] Coles, P., and Lucchin, F., Cosmology: The Origin and Evolution of Cosmic Structure, (John Wiley \& Sons, Chichester, 1996). 2.2

[37] Collar, J.I., Girard, T.A., Limagne, D., and Waysand, G., "Superheated Droplet Detectors as CDM Detectors: the SIMPLE Experiment", in Spooner, N.J.C., ed., Proc. of 1st Workshop on The Identification of Dark Matter, 563-568, (World Scientific, Singapore, 1997). 5.4.6

Living Reviews in Relativity (2002-4) http://www.livingreviews.org 
[38] Collar, J.I., Pulbasset, J., Girard, T.A., Limagne, D., Miley, H.S., and Waysand, G., "First Dark Matter Limits from a Large-Mass LowBackground Superheated Droplet Detector", Phys. Rev. Lett., 85, 30833086, (2000). For a related online version see: J.I. Collar, et al., "First Dark Matter Limits from a Large-Mass Low-Background Superheated Droplet Detector", (September, 2000), [Online Los Alamos Archive Preprint]: cited on 12 December 2000, http://xxx.lanl.gov/abs/ astro-ph/0001511. 6, 16

[39] Cooper, S., Bucci, C., von Feilitzsch, F., Höhne, J., Jochum, J., Jörgens, Loidl, M., Marchese, J., Meier, O., Meunier, P., Nagel, U., Pröbst, F., Ruloffs, A., Safran, G., Sarsa, M.L., Schnagl, J., Seidel, W., Sisti, W., Stodolsky, L., Uchaikin, S., and Zerle, L., "CRESST Dark Matter Search", in Spooner, N.J.C., and Krudryavtsev, V., eds., Proc. of 2nd Workshop on The Identification of Dark Matter, 359-364, (World Scientific, Singapore, 1999). 5.4.4, 5.4.5

[40] Damour, T., and Krauss, L.M., "A New Solar System Population of WIMP Dark Matter", Phys. Rev. Lett., 81, 5726-5729, (1998). For a related online version see: T. Damour, et al., "A New Solar System Population of WIMP Dark Matter", (1998), [Online Los Alamos Archive Preprint]: cited on 7 July 2001, http://xxx.lanl.gov/abs/astro-ph/ 9806165 3.3. 5.1

[41] de Bernadis, P., Ade, P.A.R., Bock, J.J., Bond, J.R., Borril, J., Boscaleri, A., Coble, K., Crill, B.P., De Gasperis, G., Farese, P.C., Ferreira, P.G., Ganga, K., Giacometti, M., Hivon, E., Hristov, V.V., Iocangeli, A., Jaffe, A.H., Lange, A.E., Martinis, L., Masi, S., Mason, P.V., Mauskopf, P.D., Melchiorri, A., Miglio, L., Montroy, T., Netterfield, C.B., Pascale, E., Piacentini, F., Pogosyan, D., Prunet, S., Rao, S., Romeo, G., Ruhl, J.E., Scaramuzzi, F., Sforna, D., and Vittorio, N., "A Flat Universe from High-Resolution Maps of the Cosmic Microwave Background Radiation", Nature, 404(6781), 955-959, (April, 2000). For a related online version see: P. de Bernadis, et al., "A Flat Universe from High-Resolution Maps of the Cosmic Microwave Background Radiation", (April, 2000), [Online Los Alamos Archive Preprint]: cited on 7 July 2001, http://xxx.lanl.gov/abs/astro-ph/0004404, 2.2, 2.3

[42] de Bernardis, P., Ade, P.A.R., Bock, J.J., Bond, J.R., Borrill, J., Boscaleri, A., Coble, K., Contaldi, C.R., Crill, B.P., De Troia, G., Farese, P., Ganga, K., Giacometti, M., Hivon, E., Hristov, V.V., Iacoangeli, A., Jaffe, A.H., Jones, W.C., Lange, A.E., Martinis, L., Masi, S., Mason, P., Mauskopf, P.D., Melchiorri, A., Montroy, T., Netterfield, C.B., Pascale, E., Piacentini, F., Pogosyan, D., Polenta, G., Pongetti, F., Prunet, S., Romeo, G., Ruhl, J.E., and F., Scaramuzziet, "Multiple Peaks in the Angular Power Spectrum of the Cosmic Microwave Background: Significance and Consequences for Cosmology", (May, 2001),

Living Reviews in Relativity (2002-4)

http://www. livingreviews.org 
[Online Los Alamos Archive Preprint]: cited on 7 July 2001, http: //xxx.lanl.gov/abs/astro-ph/0105296. 2.2, 2

[43] de Blok, E., McGaugh, S., and Rubin, V., "High-resolution rotation curves of LSB galaxies: Mass Models", (July, 2001), [Online Los Alamos Archive Preprint]: cited on 14 August 2001, http://xxx.lanl.gov/abs/ astro-ph/0107366. 2.3

[44] Donato, F., Fornengo, N., and Scopel, S., "Effects of Galactic Dark Halo Rotation on WIMP Direct Detection", Astropart. Phys., 9, 247260, (1998). For a related online version see: F. Donato, et al., "Effects of Galactic Dark Halo Rotation on WIMP Direct Detection", (March, 1998), [Online Los Alamos Archive Preprint]: cited on 7 July 2001, http://xxx.lanl.gov/abs/hep-ph/9803295 5.1

[45] Ellis, J., Ferstl, A., and Olive, K.A., "Exploration of Elastic Scattering Rates for Supersymmetric Dark Matter", Phys. Rev. D, 63, 065016-1065016-13, (2001). For a related online version see: J. Ellis, et al., "Exploration of Elastic Scattering Rates for Supersymmetric Dark Matter", (July, 2000), [Online Los Alamos Archive Preprint]: cited on 14 August 2000, http://xxx.lanl.gov/abs/hep-ph/0007113. 3.2, 5.1

[46] Ellis, J., Flores, R.A., and Ritz, S., "Implications for Dark Matter Particles of Searches for Energetic Solar Neutrinos", Phys. Lett. B, 198(3), 393-402, (1987). 4.1

[47] Engel, J., "Uncertainties in Neutralino-Nucleus Cross Sections", in Spooner, N.J.C., ed., Proc. of 1st Workshop on The Identification of Dark Matter, 311-317, (World Scientific, Singapore, 1997). 5.1

[48] Esposito, S., Mangano, G., Melchiorri, A., Miele, G., and Pisanti, O., "Testing Standard and Degenerate Big Bang Nucleosynthesis with BOOMERanG and MAXIMA-1", Phys. Rev. D, 63, 043004-1-043004-4, (2001). For a related online version see: S. Esposito, et al., "Testing Standard and Degenerate Big Bang Nucleosynthesis with BOOMERanG and MAXIMA-1", (July, 2000), [Online Los Alamos Archive Preprint]: cited on 14 August 2000, http://xxx.lanl.gov/abs/astro-ph/0007419. 2.3

[49] Feng, J.L., Matchev, K.T., and Wilczek, F., "Prospects for Indirect Detection of Neutralino Dark Matter", Phys. Rev. D, 63, 045024-1045024-18, (2001). For a related online version see: J.L. Feng, et al., "Prospects for Indirect Detection of Neutralino Dark Matter", (August, 2000), [Online Los Alamos Archive Preprint]: cited on 30 August 2000, http://xxx.lanl.gov/abs/astro-ph/0008115. 4.1, 7, 17

[50] Fich, M., and Tremaine, S., "The Mass of the Galaxy", Annu. Rev. Astron. Astrophys., 29, 409-45, (1991). $3.3,5$

Living Reviews in Relativity (2002-4) http://www.livingreviews.org 
[51] Fukuda, Y., et al. (For the Super-Kamiokande Collaboration, "Study of the Atmospheric Neutrino Flux in the multi-GeV Energy Range", (1998), [Online Los Alamos Archive Preprint]: cited on 14 August 2000, http: //xxx.lanl.gov/abs/hep-ex/9805006 3.2

[52] Fushimi, K., Ejiri, H., Hazama, R., Kudomi, N., Nagata, K., Ohsumi, H., Okada, K., and Tanaka, J., "Search for Exotic Nuclear Tranisition by Using the Large Volume NaI Detector of ELEGANTS V.", Nucl. Phys. $B$, 35B, 400-402, (1994). 5.2

[53] Gawiser, E., and Silk, J., "Extracting Primordial Density Fluctuations", Science, 280(5368), 1405-1411, (May, 1998). 2.2

[54] Gawiser, E., and Silk, J., "The Cosmic Microwave Background Radiation", Phys. Rep., 333, 245-267, (2000). For a related online version see: E. Gawiser, et al., "The Cosmic Microwave Background Radiation", (February, 2000), [Online Los Alamos Archive Preprint]: cited on $14 \mathrm{Au}-$ gust 2000, http://xxx.lanl.gov/abs/astro-ph/0002044 2.2

[55] Gerbier, G., Mallet, J., Mosca, L., Tao, C.and Chambon, B., Chazal, V., De Jesus, M., Drain, D., Messous, Y., and Pastor, C., "Pulse shape discrimination and dark matter search with $\mathrm{NaI}(\mathrm{Tl})$ scintillator", Astropart. Phys., 11(3), 287-302, (1999). 5.4 .2

[56] Gerhard, O., "Structure and Mass Distribution of the Milky Way Bulge and Disk", (November, 2000), [Online Los Alamos Archive Preprint]: cited on 12 December 2000, http://xxx.lanl.gov/abs/astro-ph/0010539. 3.3

[57] Ghigna, S., Moore, B., Governato, F., Lake, G., Quinn, T., and Stadel, J., "Density Profiles and Substructure of Dark Matter Halos: Converging Results at Ultra-High Numerical Resolution", Astrophys. J., 544(2), 616$628,(2000)$. For a related online version see: S. Ghigna, et al., "Density Profiles and Substructure of Dark Matter Halos: Converging Results at Ultra-High Numerical Resolution", (1999), [Online Los Alamos Archive Preprint]: cited on 7 July 2001, http://xxx.lanl.gov/abs/astro-ph/ 9910166 $2.3,3.3$

[58] Gondolo, P., Edsjo, J., Bergstrom, P., and Baltz, E.A., "DarkSUSY", (2000), [Online HTML Document]: cited on 7 July 2001, http://www. physto.se/ ${ }^{\sim}$ edsjo/darksusy/. For code download. 5.1

[59] Goobar, A., Perlmutter, S., Aldering, G., Goldhaber, G., Knop, R.A., Nugent, P., Castro, P.G., Deustua, S., Fabbro, S., Groom, D.E., Hook, I.M., Kim, A.G., Kim, M.J., Lee, J.C., Nunes, N.J., McMahon, R.G., Ruiz-Lapuente, P., Walton, N., Schaefer, B., Boyle, B.J., Filipenko, A.V., Matheson, T., Fruchtner, A.S., Panagia, N., Newberg, H.J.M., and Couch, W.J., "The Acceleration of the Universe: Measurements of Cosmological

Living Reviews in Relativity (2002-4)

http://www.livingreviews.org 
Parameters from Type Ia Supernovae", Phys. Scripta, T85, 47-58, (2000). 2.2

[60] Goodman, M.W., and Witten, E., "Detectability of certain dark-matter candidates", Phys. Rev., D31(12), 3059-3063, (1985). 5.1

[61] Governato, F., Ghigna, S., and Moore, B., "Is Cold Dark Matter Still a Strong Buy? The Lesson from Galaxy Clusters", (May, 2001), [Online Los Alamos Archive Preprint]: cited on 7 July 2001, http://xxx.lanl. gov/abs/astro-ph/0105443. Also at http://ares.merate.mi.astro. it/ fabio/. 2.3

[62] Hagmann, C., Kinion, D., Stoeffl, W., Van Bibber, K., Daw, E., Peng, H., Rosenberg, L.J., LaVeigne, J., Sikivie, P., Sullivan, N.S., Tanner, D.B., Nezrick, F., Turner, M.S., Moltz, D.M., Powlell, J., and Golubev, N.A., "Results from a High-Sensitivity Search for Cosmic Axions", Phys. Rev. Lett., 80(10), 2043-2046, (1998). 4.2

[63] Hagmann, C., Sikivie, P., Sullivan, N.S., and Tanner, D.B., "Experience with the Florida Cosmic Axion Detector", in Jones, C., and Melissinos, A., eds., Proc. Workshop on Cosmic Axions, 42-57, (World Scientific, Singapore, 1990). 4.2

[64] Halverson, N.W., Leitch, E.M., Pryke, C., Kovac, J., Carlstrom, J.E., Holzapfel, W.L., Dragovan, M., Cartwright, J.K., Mason, B.S., Padin, S., Pearson, T.J., Shepherd, M.C., and Readhead, A.C.S., "DASI First Results: A Measurement of the Cosmic Microwave Background Angular Power Spectrum", (2001), [Online Los Alamos Archive Preprint]: cited on 7 July 2001, http://xxx.lanl.gov/abs/astro-ph/0104489. 2.2

[65] Hamel, L.A., Lessard, L., Rainville, L., Zacek, V., and Sur, B., "A LowBackground Superheated Freon-Droplet Detector for Weakly-Interacting Massive Particle", in Spooner, N.J.C., ed., Proc. of 1st Workshop on The Identification of Dark Matter, 569-573, (World Scientific, Singapore, 1997). 5.4 .6

[66] Han, T., and Hempfling, R., "Messenger Sneutrinos as Cold Dark Matter", Phys. Lett. B, 415(2), 161-169, (1997). For a related online version see: T. Han, et al., "Messenger Sneutrinos as Cold Dark Matter", (1997), [Online Los Alamos Archive Preprint]: cited on 7 July 2001, http:// xxx.lanl.gov/abs/hep-ph/9708264. 3.2, 5.1

[67] Hanany, S., Ade, P., Balbi, A., Bock, J., Borrill, J., Boscaleri, A., de Bernadis, P., Ferreira, P.G., Hristov, V.V., Jaffe, A., Lange, A.E., Lee, A.T., Mauskopf, P.D., Netterfield, C.B., Oh, S., Pascale, E., Rabii, B., Richards, P.L., Smoot, G.F., Stomper, R., Winant, C.D., and Wu, J.H.P., "MAXIMA-1: A Measurement of the Cosmic Microwave Background Anisotropy on Angular Scales of $10^{\prime}-5^{\circ}$ ", Astrophys. J. Lett., 545, L5-L9, (December, 2000). For a related online version see: S. Hanany,

Living Reviews in Relativity (2002-4) http://www.livingreviews.org 
et al., "MAXIMA-1: A Measurement of the Cosmic Microwave Background Anisotropy on angular scales of 10 arcminutes to 5 degrees", (2000), [Online Los Alamos Archive Preprint]: cited on 7 July 2001, http://xxx.lanl.gov/abs/astro-ph/0005123. 2.2, 2.3

[68] Hasenbalg, F., "Annual Modulation Sensitivity in Cold Dark Matter Searches", Astropart. Phys., 9, 339-347, (1998). For a related online version see: F. Hasenbalg, "Annual Modulation Sensitivity in Cold Dark Matter Searches", (June, 1998), [Online Los Alamos Archive Preprint]: cited on 7 July 2001, http://xxx.lanl.gov/abs/astro-ph/9806198. 5.3

[69] Howard, A.S., Bewick, A., Davidge, D.C.R., Dawson, J., Jones, W.G., Lebedenko, V., Sumner, T.J., Quenby, J.J., Akimov, D.Yu., Danilov, M.V., Kovalenko, A.G., and Kovalenko, D.A., "Measurements with a TwoPhase Xenon Prototype Dark Matter Detector", in Spooner, N.J.C., and Krudryavtsev, V., eds., Proc. of 3rd Workshop on The Identification of Dark Matter, 457-462, (World Scientific, Singapore, 2001). 5.4.3

[70] Irastorza, I.G., Morales, A., Aalseth, C.E., Avignone III, F.T., Brodzinski, R.L., Cebrian, S., Garcia, E., Gonzalez, D., Hensley, W.K., Miley, H.S., Morales, J., Ortiz de Solorzano, A., Puimedon, J., Reeves, J.H., Sarsa, M.L., Scopel, S., and Villar, J.A., "Recent Results from the CANFRANC Dark Matter Search using Germanium Detectors", (November, 2000), [Online Los Alamos Archive Preprint]: cited on 12 December 2000, http://xxx.lanl.gov/abs/hep-ph/0011318. 16

[71] Kajita, T., and the Super-KAMIOKANDE Collaboration, "Atmospheric Neutrino Observation in Super-Kamiokande - Evidence for $\nu_{\mu}$ Oscillations", in Minakata, H., and Yasuda, O., eds., Proc. Neutrino-98, 107, (Universal Academy Press, Tokyo, 1998). 3.2

[72] Kalberla, P.M.W., and Kerp, J., "Hydrostatics of the Galactic Halo", in Gibson, B.K., Axelrod, T.S., and Putman, M.E., eds., The Third Stromlo Symposium: The Galactic Halo, volume 165 of ASP Conf. Ser., 453, (ASP, San Francisco, 1999). For a related online version see: P.M.W. Kalberla, et al., "Hydrostatics of the Galactic Halo", (1998), [Online Los Alamos Archive Preprint]: cited on 7 July 2001, http://xxx.lanl.gov/ abs/astro-ph/9811439. 3.3 .5

[73] Kamionkowski, M., and A., Kinkhabwala, "Galactic Halo Models and Particle Dark Matter Detection", Phys. Rev. D, 57, 3256-3263, (1998). For a related online version see: M. Kamionkowski, et al., "Galactic Halo Models and Particle Dark Matter Detection", (October, 1997), [Online Los Alamos Archive Preprint]: cited on 7 July 2001, http://xxx.lanl. gov/abs/hep-ph/9710337. 5.1

Living Reviews in Relativity (2002-4)

http://www.livingreviews.org 
[74] Kim, J.E., "A Theoretical Review of Axion", (February, 2000), [Online Los Alamos Archive Preprint]: cited on 14 August 2000, http://xxx. lanl.gov/abs/astro-ph/0002193. 3.2

[75] Kim, J.E., "Axion Theory Review", in Spooner, N.J.C., and Krudryavtsev, V., eds., Proc. of 2nd Workshop on The Identification of Dark Matter, 248-260, (World Scientific, Singapore, 1999). 4.2

[76] Klapdor-Kleingrothaus, H.V., "New Underground Neutrino Observatory - GENIUS - In the New Millenium: For Solar Neutrinos, Dark Matter and Double Beta Decay", (April, 2001), [Online Los Alamos Archive Preprint]: cited on 7 July 2001, http://xxx.lanl.gov/abs/hep-ph/0104028. 7

[77] Lee, A.T., Ade, P., Balbi, A., Bock, J., Borrill, J., Boscaleri, A., De Bernardis, P., Ferreira, P.G., Hanany, S., Hristov, V.V., Jaffe, A.H., Mauskopf, P.D., Netterfield, C.B., Pascale, E., Rabii, B., Richards, P.L., Smoot, G.F., Stompor, R., Winant, C.D., and J.H.P., Wu, "A High Spatial Resolution Analysis of the MAXIMA-1 Cosmic Microwave Background Anisotropy Data", Astrophys. J., 561, L1-L6, (2001). For a related online version see: A.T. Lee, et al., "A High Spatial Resolution Analysis of the MAXIMA-1 Cosmic Microwave Background Anisotropy Data", (2001), [Online Los Alamos Archive Preprint]: cited on 7 July 2001, http://xxx.lanl.gov/abs/astro-ph/0104459 2.2

[78] Lewin, J.D., and Smith, P.F., "Review of Mathematics, Numerical Factors, and Corrections for Dark Matter Experiments Based on Elastic Nuclear Recoil", Astropart. Phys., 6, 87-112, (1996). 3.3

[79] Mandic, V., Gaitskell, R., and Golwala, S., "Minimal Supersymmetry, Supersymmetric Frameworks, and Expected Neutralino-Quark Scattering Cross-Section", http://cfpa.berkeley.edu/ cdmsuser/limitplots/, (1999). 3.2, 5.1, 6

[80] Marchesini, D., D’Onghia, E., Chincarini, G., Firmani, C., Conconi, P., Molinari, E., and Zacchei, A., "Soft Cores in Late-Type Dwarf and LSB Galaxies from H $\alpha$ Observations", (July, 2001), [Online Los Alamos Archive Preprint]: cited on 7 August 2001, http://xxx.lanl.gov/abs/ astro-ph/0107424. 2.3

[81] McGaugh, S.S., "BOOMERanG Data Suggest a Purely Baryonic Universe", Astrophys. J., 541, L33-L36, (2000). For a related online version see: S.S. McGaugh, "BOOMERanG Data Suggest a Purely Baryonic Universe", (August, 2000), [Online Los Alamos Archive Preprint]: cited on 12 December 2000, http://xxx.lanl.gov/abs/astro-ph/0008188, 2.3

[82] McGaugh, S.S., and de Blok, W.J.G., "Testing the Hypothesis of Modified Dynamics with Low Surface Brightness Galaxies and Other Evidence", Astrophys. J., 499(1), 66-81, (1998). 2.3 
[83] Meikle, W.P.M.S., "The Absolute Infrared Magnitudes of Type Ia Supernovae", Mon. Not. R. Astron. Soc., 314, 782-792, (2000). 2.3

[84] Melissinos, A.C. et al., "Experience with the Rochester-BrookhavenFermilab Axion Detector", in Jones, C., and Melissinos, A., eds., Proc. Workshop on Cosmic Axions, 39-41, (World Scientific, Singapore, 1990). 4.2

[85] Milgrom, M., "The Shape of Dark Matter Halos in Disc Galaxies According to the Modified Dynamics", (2001), [Online Los Alamos Archive Preprint]: cited on 7 July 2001, http://xxx.lanl.gov/abs/astro-ph/ 0101227. 2.3

[86] Milgrom, M., "A Modification of the Newtonian Dynamics: Implications for Galaxies", Astrophys. J., 270(2), 371-383, (1983). 2.3

[87] Minowa, M., Moriyama, S., Inoue, Y., Namba, T., Takasu, Y., and Yamamoto, A., "The Tokyo Axion Helioscope Experiment", in Spooner, N.J.C., and Krudryavtsev, V., eds., Proc. of 2nd Workshop on The Identification of Dark Matter, 447-453, (World Scientific, Singapore, 1999). 4.2

[88] Mohr, J.J., Mathiesen, B., and Evrard, A.E., "Properties of the Intracluster Medium in an Ensemble of Nearby Galaxy Clusters", Astrophys. J., 517(2), 627-649, (June, 1999). For a related online version see: J.J. Mohr, et al., "Properties of the Intracluster Medium in an Ensemble of Nearby Galaxy Clusters", (1999), [Online Los Alamos Archive Preprint]: cited on 7 July 2001, http://xxx.lanl.gov/abs/astro-ph/9901281. 3.1

[89] Moiseev, A., "Detection of Galactic Dark Matter by GLAST", in Kieda, D., Salamon, M., and Dingus, B., eds., Proc. $26^{\text {th }}$ International Cosmic Ray Conference, Proc. 26th International Cosmic Ray Conference, 2:2732:276, (University of Utah, Utah, 1999). 7

[90] Moore, B., "The N-Body Site", (August, 2000), [Online HTML Document]: cited on 30 August 2000, http://star-www.dur.ac.uk/ ${ }^{\sim m o o r e / / . ~}$ $2.3,4,3.3$

[91] Moore, B., "The Dark Matter Crisis", (March, 2001), [Online Los Alamos Archive Preprint]: cited on 14 June 2001, http://xxx.lanl.gov/abs/ astro-ph/0103100. 2.3

[92] Moore, B., Calcaneo-Roldan, C., Stadel, J., Quinn, T., Lake, G., Ghigna, S., and Governato, F., "Dark Matter in Draco and the Local Group: Implications for Direct Detection Experiments", (June, 2001), [Online Los Alamos Archive Preprint]: cited on 30 June 2001, http://xxx. lanl.gov/abs/astro-ph/0106271. 3.3

[93] Moskowitz, B.E., "Cosmic Axion Searches", Nucl. Instrum. Methods, A264(1), 98-105, (1988). 4.2

Living Reviews in Relativity (2002-4)

http://www.livingreviews.org 
[94] Navarro, J.F., Frenk, C.S., and White, S.D.M., "The Structure of Cold Dark Matter Halos", Astrophys. J., 462(2), 563-575, (1996). 2.2, 2.3

[95] Navarro, J.F., Frenk, C.S., and White, S.D.M., "An Universal Density Profile from Hierarchical Clustering", Astrophys. J., 490(2), 493-508, (1997). 2.2, 2.3

[96] Navarro, J.F., and Steinmetz, M., "Dark Halo and Disk Galaxy Scaling Laws in Hierarchical Universes", (January, 2000), [Online Los Alamos Archive Preprint]: cited on 4 January 2000, http://xxx.lanl.gov/abs/ astro-ph/0001003. 2.3

[97] Netterfield, C.B., Ade, P.A.R., Bock, J.J., Bond, J.R., Borrill, J., Boscaleri, A., Coble, K., Contaldi, C.R., Crill, B.P., de Bernadis, P., Farese, P., Ganga, K., Giacometti, M., Hivon, E., Hristov, V.V., Iacoangeli, A., Jaffe, A.H., Jones, W.C., Lange, A.E., Martinis, L., Masi, S., Mason, P., Mauskopf, P.D., Melchiorri, A., Montroy, T., Pascale, E., Piacentini, F., Pogosyan, D., Pongetti, F., Prunet, S., Romeo, G., Ruhl, J.E., and Scaramuzzi, F., "A Measurement by BOOMERANG of Multiple Peaks in the Angular Power Spectrum of the Cosmic Microwave Background", (2001), [Online Los Alamos Archive Preprint]: cited on 7 July 2001, http://xxx.lanl.gov/abs/astro-ph/0104460 2.2

[98] Ni, W-T., Pan, S-S., Yeh, H-C., Hou, L-S., and Wan, J., "Search for Anomalous Spin-Dependent Forces Using a DC SQUID with a Paramagnetic Salt", in Jantzen, R.T., Keiser, M., and Ruffini, R., eds., Proceedings of the 7th Marcel Grossman Meeting, 1625, (World Scientific, New Jersey, 1996). 4.2

[99] Oort, J.H., "The Force Exerted by the Stellar System in the Direction Perpendicular to the Galactic Plane and Some Related Problems", Bull. Astron. Inst. Neth., 6, 249, (1932). 2.1

[100] Oort, J.H., "Note on the Determination of $K_{Z}$ and on the Mass Density Near the Sun", Bull. Astron. Inst. Neth., 15, 45, (1960). 2.1

[101] Ovchinnikov, B.M., and Parusov, V.V., "A Method for Background Reduction in an Experiment for WIMP Search with a Xe(Ar)-Liquid Ionisation Chamber", Astropart. Phys., 10, 129-132, (1999). 5.4.3

[102] Padin, S., Cartwright, J.K., Mason, B.S., Pearson, T.J., Readhead, A.C.S., Sheperd, M.C., Sievers, J., Udomprasert, P.S., Holzapfel, W.L., Myers, S.T., Carlstrom, J.E., Leitch, E.M., Bronfman, L., and May, J., "First intrinsic anisotropy observations with the Cosmic Background Imager", Astrophys. J. Lett., 549(1), L1-L5, (2001). 2.2

[103] Persic, M., and Salucci, P., "The Baryon Content of the Universe", Mon. Not. R. Astron. Soc., 258(1), 14-18, (1992). 3 
[104] Pfenniger, D., Combes, F., and Martinet, L., "Is Dark-Matter in Spiral Galaxies Cold Gas .1. Observational Constraints and Dynamical Clues About Galaxy Evolution", Astron. Astrophys., 285(1), 79-93, (1994). 3.1

[105] Primack, J.R., Seckel, D., and Sadoulet, B., "Detection of Cosmic Dark Matter", Annu. Rev. Nucl. Part. S., 38, 751-807, (1988). 4

[106] Quenby, J.J., Sumner, T.J., Li, J.P., Bewick, A., Grant, S.M., Shaul, D., Smith, N.J.T., Jones, W.G., Davies, G.J., Zammit, C.C., Caplin, A.D., Stradling, R.A., Alt, T., Lally, C.H., Smith, P.F., Homer, G.J., Arnison, G.T.J., Lewin, J.D., Alner, G.J., Cruise, A.M., Vandenputte, M.J.J., Spooner, N.J.C., Barton, J.C., Blake, P.R., Lea, M.J., Stefanyi, P., and Saunders, J., "Results from the First Stage of a UK Galactic Dark Matter Search Using a Low Background Sodium Iodide Detector", Phys. Lett. B, 351, 70-76, (1995). 5.4.2, 10

[107] Ressell, M.T., and Dean, D.J., "The Spin-Dependent Neutralino-Nucleus Form Factor for ${ }^{127}$ I", in Spooner, N.J.C., ed., Proc. of 1st Workshop on The Identification of Dark Matter, 318-323, (World Scientific, Singapore, 1997). 5.1

[108] Riess, A.G., Nugent, P.E., Schmidt, B.P., Tonry, J., Dickinson, M., Gilliland, R.L., Thompson, R.I., Budavari, T., Casertano, S., Evans, A.S., Filippenko, A.V., Livio, M., Sanders, D.B., Shapley, A.E., Spinrad, H., Steidel, C.C., Stern, D., Surace, J., and Veilleux, S., "The Farthest Known Supernova: Support for an Accelerating Universe and a Glimpse of the Epoch of Deceleration", Astrophys. J., 560, 49-71, (2001). For a related online version see: A.G. Riess, et al., "The Farthest Known Supernova: Support for an Accelerating Universe and a Glimpse of the Epoch of Deceleration", (April, 2001), [Online Los Alamos Archive Preprint]: cited on 7 July 2001, http://xxx.lanl.gov/abs/astro-ph/0104455. 2.3

[109] Rosenberg, L.J., "Invisible Axions: Overview of Experiments", in Spooner, N.J.C., and Krudryavtsev, V., eds., Proc. of 2nd Workshop on The Identification of Dark Matter, 261-274, (World Scientific, Singapore, 1999). 4.2

[110] Roszkowski, L., "Light Neutralinos as Dark Matter", Phys. Lett. B, 262, $59-67,(1991) .5 .1$

[111] Roszkowski, L., "Neutralino Dark Matter: Status and Prospects", in International Workshop on Non-Accelerator New Physics, (1997). 5.1

[112] Salucci, P., and Borriello, A., "The Distribution of Dark Matter in Galaxies: Constant- Density Dark Halos Envelop the Stellar Disks", (2000), [Online Los Alamos Archive Preprint]: cited on 12 December 2000, http://xxx.lanl.gov/abs/astro-ph/0011079. 2.3

Living Reviews in Relativity (2002-4)

http://www.livingreviews.org 
[113] Saunders, W., Sutherland, W.J., Maddox, S.J., Keeble, O., Oliver, S.J., Rowan-Robinson, M., McMahon, R.G., Efstathiou, G.P., Tadros, H., White, S.D.M., Frenk, C.S., Carraminana, A., and Hawkins, M.R.S., "The PSCz Catalogue", Mon. Not. R. Astron. Soc., 317, 55-64, (2000). For a related online version see: W. Saunders, et al., "The PSCz Catalogue", (2000), [Online Los Alamos Archive Preprint]: cited on 7 July 2001, http://xxx.lanl.gov/abs/astro-ph/0001117. 2.2

[114] Shaul, D.A., Sumner, T.J., Rochester, G.K., and Speake, C.C., "On the Calculation of Spin-Coupling Forces", in Sumner, T.J., ed., Fundamental Physics in Space, Class. Quant. Gravity 13, A107-A112, (IOP, Bristol, 1996). 4.2

[115] Sikivie, P., and Kinney, W., "Dark Matter Caustics", (April, 2000), [Online Los Alamos Archive Preprint]: cited on 14 August 2000, http: //xxx.lanl.gov/abs/astro-ph/0004356. 3.3

[116] Sikivie, P., Tkachev, I.I., and Wang, Y., "Secondary Infall Model of Galactic Halo Formation and the Spectrum of Cold Dark Matter Particles on Earth", Phys. Rev., D56(4), 1863-1878, (1997). 3.3, 5.1

[117] Smith, P.F., "Comparison of Recoil Discrimination Potential in Sodium Iodide, Liquid Xenon and Gaseous Track Detectors", in Spooner, N.J.C., ed., Proc. of 1st Workshop on The Identification of Dark Matter, 335-344, (World Scientific, Singapore, 1997). 5.4 .313

[118] Smith, P.F., Arnison, G.T.J., Homer, G.J., Lewin, J.D., Alner, G.J., Spooner, N.J.C., Quenby, J.J., Summer, T.J., Bewick, A., Li, P., Shaul, D., Ali, T., Jones, W.G., Smith, N.J.T., Davies, G.J., Lally, C.H., van den Putte, M.J., Barton, J.C., and Blake, P.R., "Improved Dark Matter Limits from Pulse Shape Discrimination in a Low Background Sodium Iodide Detector at the Boulby Mine UK Underground NaI Detector", Phys. Lett. $B, \mathbf{3 7 9}, 299-308,(1996)$. $5.4 .2,10$

[119] Smith, P.F., and Lewin, J.D., "Dark Matter Detection", Phys. Rep., 187, 203-280, (1990). 4, 4.2

[120] Smith, P.F., Smith, N.J.T., Lewin, J.D., Homer, G.J., Alner, J., Arnison, G.T.J., Quenby, J.J., Sumner, T.J., Bewick, A., Ali, T., Ahmed, B., Howard, A.S., Davidge, D., Joshi, M., Jones, W.G., Davies, G., Lehner, M.J., McMillan, J.E., Peak, C.D., Kudryatsev, V.A., and Barton, J.C., "Dark Matter Experiments at the UK Boulby Mine", Phys. Rep., 307, 275-282, (1998). 6

[121] Sonnenschein, A., Bauer, D.A., Caldwell, D.O., Nelson, H., Yellin, S., Brink, P.L., Cabrera, B., Clarke, R.M., Colling, P., Davies, A.K., Irwin, K.D., Nam, S.W., Penn, M.J., Akerib, D.S., Bolodyaynya, A., Perera, T.A., Schnee, R.W., Crisler, M.B., Dixon, R., Eichblatt, S., da Silva, A., Gaitskell, R.J., Golwala, S.R., Jochum, J., Barnes, P.D., Sadoulet,

Living Reviews in Relativity (2002-4) http://www.livingreviews.org 
B., Shutt, T., Spadafora, A.L., Stockwell, W.K., Haller, E.E., Knowlton, W.B., Ross, R.R., Smith, A., Smith, G., Young, B.A., Neuhausser, B., and Lipschultz, F., "Results of the Cryogenic Dark Matter Search (CDMS) Obtained with Thermistor-Instrumented Germanium Calorimeters", in Spooner, N.J.C., and Krudryavtsev, V., eds., Proc. of 2nd Workshop on The Identification of Dark Matter, 347-352, (World Scientific, Singapore, 1999). 5.4 .5

[122] Speake, C.C., Hammond, G., Trenkel, C., Rochester, G.K., and Sumner, T.J., "A Preliminary Study of a Torsion Balance Based on a Spherical Superconducting Suspension", Meas. Sci. Technol., 10, 508-513, (1999). 4.2

[123] Speake, C.C., and Leon, J., "New Possible Detection Schemes for Relic Galactic Neutrinos", in Sumner, T.J., ed., Fundamental Physics in Space, Class. Quant. Gravity 13, A207-A218, (IOP, Bristol, 1996). 4.2

[124] Spergel, D.N., and Press, W.H., "Effect of Hypothetical, Weakly Interacting Massive Particles on Energy Transport in the Solar Interior", Astrophys. J., 294, 663-673, (1985). 3.3

[125] Spooner, N.J.C., Kudryavtsev, V.A., Lawson, T.B., Lehner, M.J., Lightfoot, P.K., McMillan, J.E., Peak, C.D., Roberts, J.W., Tovey, D.R., Ward, C.K., Smith, P.F., Smith, N.J.T., Lewin, J.D., Homer, G.J., Alner, G.J., Arnison, G.T.J., Sumner, T.J., Quenby, J.J., Jones, W.G., Ahmed, B., Ali, T., Bewick, A., Davidge, D., Davies, G.J., Howard, A.S., Joshi, M., Liubarsky, I., Smith, R.A.D., and Barton, J.C., "Searches for Dark Matter at Boulby Mine - Investigation of Anomalous Events in NaI", in Spooner, N.J.C., and Krudryavtsev, V., eds., Proc. of 2nd Workshop on The Identification of Dark Matter, 307-315, (World Scientific, Singapore, 1999). 6

[126] Spooner, N.J.C., Roberts, J.W., and Tovey, D.R., "Measurements of Carbon Recoil Scintillation Efficiency and Anisotropy in Stilbene for WIMP Searches with Directional Sensitivity", in Spooner, N.J.C., ed., Proc. of 1st Workshop on The Identification of Dark Matter, 481-486, (World Scientific, Singapore, 1997). 5.4 .2

[127] Spooner, N.J.C., and Smith, P.F., "The UVIS Scintillation Detector A Proposed Method of Nuclear Recoil Discrimination for Dark Matter Searches", Phys. Lett. B, 314, 430-435, (1995). 5.4 .2

[128] Spooner, N.J.C., Tovey, D.R., Peak, C.D., and Roberts, J., "Demonstration of Nuclear Recoil Discrimination Using Recoil Range in a Mixed $\mathrm{CaF}_{2}$ + Liquid Scintillator Gel Detector for Dark Matter Searches", Astropart. Phys., 8, 13-19, (1997). 5.4 .2

[129] Stecker, F.W., "Cosmic Gamma-Rays from Dark Matter and Antimatter Annihilation", Nucl. Phys., 10B, 93-107, (1989). 4.1

Living Reviews in Relativity (2002-4)

http://www.livingreviews.org 
[130] Stecker, F.W., and Tylka, A.J., "Spectra, Fluxes, and Observability of Gamma Rays from Dark Matter Annihilation in the Galaxy", Astrophys. J., 343, 169-176, (1989). 4.1

[131] Sumner, T.J., "The Search for Non-baryonic Dark Matter", in Blake, P.R., and Nash, W.F., eds., Solar and Galactic Cosmic Rays, Nucl. Phys. B (Proc. Suppl.) 22B, 165-177, (Elsevier (North-Holland), Amsterdam, 1991). 4

[132] Sumner, T.J., "Detection of Weakly Interacting Massive Particles in the Halo of Our Galaxy: Techniques Above $\sim 100$ K", in Spooner, N.J.C., and Krudryavtsev, V., eds., Proc. of 2nd Workshop on The Identification of Dark Matter, 227-240, (World Scientific, Singapore, 1999). 4.2

[133] Sumner, T.J., "Prospects for a Large Xe Detector", in Spooner, N.J.C., and Krudryavtsev, V., eds., Proc. of 3rd Workshop on The Identification of Dark Matter, 603-605, (World Scientific, Singapore, 2001). 7

[134] Sumner, T.J., Speake, C.C., Rochester, G.K., Leon, J., and Jafry, Y., "Satellite Search for Psuedo-scalar Interactions, SSPIN", Propsal to European Space Agency for F2/F3, (2000). 4.2

[135] Sumner, T.J. (For the UKDMC-ITEP-Columbia-UCLA-TorinoCERN collaboration), "ZEPLIN-III: A Two-Phase Xenon Dark Matter Detector", in Spooner, N.J.C., and Krudryavtsev, V., eds., Proc. of 3rd Workshop on The Identification of Dark Matter, 452-456, (World Scientific, Singapore, 2001). 5.4 .3

[136] Tegmark, M., "Max Tegmark's library: concordance", (August, 2000), [Online HTML Document]: cited on 30 August 2000, http://www.hep. upenn.edu/ ${ }^{\sim} \max /$ concordance.html. 2.2

[137] Tegmark, M., Zaldarriaga, M., and Hamilton, A.J.S., "Latest Cosmological Comstraints on the Densities of Hot and Cold Dark Matter", (August, 2000), [Online Los Alamos Archive Preprint]: cited on 14 August 2000, http://xxx.lanl.gov/abs/hep-ph/0008145. 2.2, 1

[138] Turner, M.S., "Dark Matter, Dark Energy, and Fundamental Physics", (December, 1999), [Online Los Alamos Archive Preprint]: cited on 9 December 1999, http://xxx.lanl.gov/abs/astro-ph/9912211. 3

[139] Turner, M.S., "Windows on the Axion", Phys. Rep., 197, 69, (1990). 4.2

[140] Walker, M.A., "Unambiguous Quasar Microlensing", Mon. Not. R. Astron. Soc., 306(2), 504-508, (1999). 3.1

[141] Walker, M.A., and Wardle, M., "Exteme Scattering Events and Galactic Dark Matter", Astrophys. J., 498(2), L125-L128, (1998). 3.1

Living Reviews in Relativity (2002-4) http://www.livingreviews.org 
[142] Wang, H., "Xenon as a Detector for Dark Matter Search", Phys. Rep., 307, 263-267, (1998). 5.4 .3

[143] Wang, X., Tegmark, M., and Zaldarriaga, M., "Is cosmology consistent?", (May, 2001), [Online Los Alamos Archive Preprint]: cited on 7 July 2001, http://xxx.lanl.gov/abs/astro-ph/0105091. 1, 2.2, 1, 2, 2.3

[144] Weinberg, D.H., Miralda-Escude, J., Hernquist, L., and Katz, N., "A Lower Bound of the Cosmic Baryion Density", Astrophys. J., 490(2), 564-570, (1997). 3.1

[145] Yamamoto, K., and Matsuki, S., "Quantum Analysis of Rydberg Atom Cavity Detector for Dark Matter Axion Search", in Spooner, N.J.C., and Krudryavtsev, V., eds., Proc. of 2nd Workshop on The Identification of Dark Matter, 474-479, (World Scientific, Singapore, 1999). 4.2 